ARTICLE

\title{
Altered function and differentiation of age-associated $B$ cells contribute to the female bias in lupus mice
}

Edd Ricker ${ }^{1,2}$, Michela Manni ${ }^{1}$, Danny Flores-Castro', Daniel Jenkins ${ }^{1}$, Sanjay Gupta1 , Juan Rivera-Correa ${ }^{1,2}$, Wenzhao Meng ${ }^{3}$, Aaron M. Rosenfeld (10 ${ }^{3}$, Tania Pannellini ${ }^{4}$, Mahesh Bachu ${ }^{5}$, Yurii Chinenov ${ }^{6}$, Peter K. Sculco ${ }^{7}$, Rolf Jessberger (1) ${ }^{8}$, Eline T. Luning Prak (1) ${ }^{3} \&$ Alessandra B. Pernis (1) $1,2,6,9 凶$

Differences in immune responses to viruses and autoimmune diseases such as systemic lupus erythematosus (SLE) can show sexual dimorphism. Age-associated $B$ cells ( $A B C$ ) are a population of $\mathrm{CD} 11 \mathrm{c}^{+} \mathrm{T}$-bet ${ }^{+} \mathrm{B}$ cells critical for antiviral responses and autoimmune disorders. Absence of DEF6 and SWAP-70, two homologous guanine exchange factors, in doubleknock-out (DKO) mice leads to a lupus-like syndrome in females marked by accumulation of $A B C$ s. Here we demonstrate that DKO ABCs show sex-specific differences in cell number, upregulation of an ISG signature, and further differentiation. DKO ABCs undergo oligoclonal expansion and differentiate into both $\mathrm{CD}_{11 \mathrm{c}^{+}}$and $\mathrm{CD} 11 \mathrm{c}^{-}$effector $\mathrm{B}$ cell populations with pathogenic and pro-inflammatory function as demonstrated by BCR sequencing and fatemapping experiments. TIr7 duplication in DKO males overrides the sex-bias and further augments the dissemination and pathogenicity of $A B C s$, resulting in severe pulmonary inflammation and early mortality. Thus, sexual dimorphism shapes the expansion, function and differentiation of $A B C s$ that accompanies TLR7-driven immunopathogenesis.

\footnotetext{
${ }^{1}$ Autoimmunity and Inflammation Program, Hospital for Special Surgery, New York, NY, USA. ${ }^{2}$ Department of Microbiology and Immunology, Weill Cornel Medicine, New York, NY, USA. ${ }^{3}$ Department of Pathology and Laboratory Medicine, Perelman School of Medicine, Philadelphia, PA, USA. ${ }^{4}$ Research Division and Precision Medicine Laboratory, Hospital for Special Surgery, New York, NY, USA. ${ }^{5}$ Arthritis and Tissue Degeneration Program, Hospital for Special Surgery, New York, NY, USA. ${ }^{6}$ David Z. Rosensweig Genomics Research Center, Hospital for Special Surgery, New York, NY, USA. ${ }^{7}$ Department of Orthopedic Surgery, Hospital for Special Surgery, New York, NY, USA. ${ }^{8}$ Institute of Physiological Chemistry, Technische Universitat, Dresden, Germany.

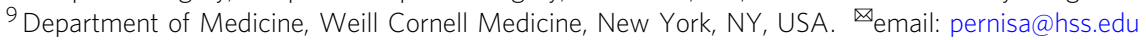


S ex-dependent differences in immune responses have been well documented in viral infections, vaccination outcomes, and autoimmune diseases like Systemic Lupus Erythematosus (SLE), a heterogeneous disorder that often includes upregulation of interferon stimulated genes (ISG) in addition to autoantibody production and multi-organ involvement ${ }^{1,2}$. Both sex hormones and genes on the X-chromosome have been implicated in this sexual dimorphism ${ }^{3}$. Notably, TLR7, an endosomal TLR critical for responses to viruses like SARS-CoV-2 and lupus pathogenesis, is encoded on the $\mathrm{X}$-chromosome and has recently been shown to partially escape X-chromosome inactivation resulting in greater TLR7 expression in a proportion of female B cells, monocytes, and pDCs ${ }^{4}$.

TLR7 engagement promotes the formation of Age/autoimmune-associated $\mathrm{B}$ cells (ABC), also known as $\mathrm{DN} 2$ in humans, a B cell population that preferentially expands with age in female mice ${ }^{5-7}$. ABCs exhibit a distinctive phenotype and, in addition to classical $\mathrm{B}$ cell markers, express the transcription factor T-bet and myeloid markers like CD11c. T-bet and CD11c are often, but not always, co-expressed ${ }^{8-10}$. ABCs are an important component of antiviral responses and are inappropriately controlled in several viral infections including HIV and SARS$\mathrm{Co}-\mathrm{V} 2^{11-13}$. Aberrant expansion and activation of ABCs is also associated with autoimmune pathogenesis, especially SLE ${ }^{14}$. In this disease, $\mathrm{ABCs}$ accumulate to a greater extent in AfricanAmerican patients, rapidly differentiate into plasmablasts/plasma cells $(\mathrm{PB} / \mathrm{PC})$, are major producers of autoantibodies, and correlate with disease activity and clinical manifestations $7,15,16$. Despite the emerging biological and clinical importance of $\mathrm{ABCs}$, the full spectrum of their function and differentiation capabilities are incompletely understood.

Although T-bet is a well-known marker for ABCs, reliance of $A B C s$ on this transcription factor differs depending on the setting. B-cell T-bet is important for protective flu-specific IgG2c antibodies, but its absence has variably impacted the generation of $\mathrm{ABCs}$ and disease parameters in lupus murine models ${ }^{9,17-19}$. This is likely due to the presence of additional regulators of autoimmune ABCs such as IRF5 (Interferon Regulatory Factor 5), whose dysregulation promotes $\mathrm{ABC}$ accumulation and lupus development ${ }^{20}$. The ability of IRF5 to drive ABC expansion can be restrained by the SWEF proteins, Def6 and SWAP-70, two homologous proteins that also control cytoskeletal reorganization by regulating Rho GTPase signaling and whose combined absence in mice results in a lupus syndrome that primarily affects females ${ }^{21-24}$. An important role for these molecules in immune responses, inflammation, and autoimmunity is supported not only by murine genetic models but also by human studies. The CORO1A-DEF6 blood transcription module correlates with responses to flu vaccination and malaria 25,26 . Furthermore, SWAP70 is a susceptibility locus for $\mathrm{RA}^{27}$ and $\mathrm{CVD}^{28}$ while DEF6 is a risk factor for human SLE ${ }^{29,30}$. Mutations in DEF6 moreover result in early-onset autoimmune manifestations, often associated with viral infections, which include autoantibody production and upregulation of an ISG signature 31,32 .

In this study we have exploited the sex-bias exhibited by mice lacking both SWEF proteins, DEF6 and SWAP-70 (DoubleKnock-out or DKO) to investigate the impact of sexual dimorphism on $\mathrm{ABC}$ function. We show that $\mathrm{ABCs}$ from $\mathrm{DKO}$ females and males differ in their ability to expand, upregulate an ISG signature, and further differentiate. BCR sequencing and fate mapping experiments indicate marked oligoclonal expansion and interrelatedness of $\mathrm{ABCs}$ with both $\mathrm{CD} 11 \mathrm{c}^{+}$and $\mathrm{CD} 11 \mathrm{c}^{-}$effector populations, which include $\mathrm{CD} 11 \mathrm{c}^{+}$pre-GC B cells and $\mathrm{CD} 11 \mathrm{c}^{+}$ PBs. In addition to IRF5, DKO ABCs also require IRF8 but are less dependent on T-bet. Notably, Tlr7 duplication in DKO males overrides the sex-bias and augments the pathogenicity of $\mathrm{ABCs}$ resulting in severe pathology and early mortality. Thus, in autoimmune settings, $\mathrm{ABCs}$ can give rise to a heterogenous population of effector cells with distinct pathogenic potentials that are controlled in a sexually dimorphic manner.

\section{Results}

$\mathrm{ABC}$ accumulation and function in DKOs is sex-dependent and controlled by TLR7. Similar to human SLE, the lupus syndrome that develops in DKOs preferentially affects females providing a powerful model to delineate the cellular and molecular mechanisms that underlie sexual dimorphism in autoimmunity. Given the key role of ABCs in lupus, we first assessed whether the sex-bias that accompanies lupus development in DKOs was associated with differences in $A B C$ expansion. Significantly more ABCs accumulated in DKO females than age-matched DKO males, although DKO males still contained greater numbers of ABCs than WT controls (Fig. 1a; Supplementary Fig. 1A). Furthermore, $\mathrm{ABCs}$ sorted from DKO males secreted significantly lower levels of anti-dsDNA IgG2c upon stimulation with a TLR7 agonist, imiquimod, than ABCs from DKO females (Fig. 1b). Thus, both the accumulation and the function of ABCs in DKOs are controlled in a sex-specific manner.

$T l r 7$ can be expressed biallelically in a proportion of female B cells due to incomplete $\mathrm{X}$ chromosome inactivation ${ }^{4}$. In line with these findings, $\mathrm{ABCs}$ from $\mathrm{DKO}$ females expressed higher levels of $T l r 7$ than ABCs from DKO males (Supplementary Fig. 1B). $A B C$ accumulation in DKO females was furthermore dependent on TLR7, as DKO females crossed to Tlr $7^{-1-}$ mice exhibited a profound reduction in $\mathrm{ABC}$ accumulation (Fig. 1c). Tlr7-deficient DKO females also displayed significant decreases in GC B cells, $\mathrm{CD} 138^{+}$plasmablasts/plasma cells (PB/PCs), and $\mathrm{T}_{\mathrm{FH}}$ cells, and lacked anti-dsDNA IgG2c antibodies (Supplementary Fig. 1C-F). Thus, $\mathrm{ABC}$ expansion and lupus pathogenesis in DKO females are dependent on Tlr7.

To further assess the importance of Tlr7 in the sex-bias of DKOs, we crossed DKO males to C57BL/6 mice carrying the Ylinked genomic modifier Yaa (termed Yaa-DKOs), in which a portion of the X-chromosome has translocated onto the Ychromosome resulting in a 2-fold increase in Tlr7 expression in males ${ }^{33}$. Tlr 7 duplication in DKO males markedly increased the frequencies and numbers of splenic $\mathrm{ABCs}$ reaching levels that were even greater than those observed in DKO females (Fig. 1d; Supplementary Fig. 1F). Tlr7 duplication in DKO males also rescued the ability of sorted male $\mathrm{ABCs}$ to secrete anti-dsDNA IgG2c antibodies upon stimulation (Fig. 1e). Increased ABC accumulation and function in Yaa-DKO males were accompanied by autoantibody production, the classical clinical feature of SLE (Fig. 1f, g). Total antibody titers were also comparable between DKO females and Yaa-DKO males (Supplementary Fig. 1H). Yaa-DKO males also exhibited significantly decreased survival as compared to both DKO males and females (Fig. 1h). Thus, duplication of $T \operatorname{Tr} 7$ in Yaa-DKO males overrides the sex-bias and promotes the development of a severe lupus syndrome in DKO males marked by greatly enhanced accumulation of $\mathrm{ABCs}$ and autoantibody responses.

The expansion of GC B cells and PB/PCs in DKOs is regulated in a sex-specific manner. In addition to $A B C$ accumulation, DKO females also exhibit robust GC and PB/PC responses ${ }^{22}$, prompting us to examine whether sex-specific differences could also be observed in these compartments. DKO females contained more $\mathrm{GL} 7{ }^{+} \mathrm{Fas}^{+} \mathrm{GC}$ B cells than DKO males, a difference that was again reversed by $T l r 7$ duplication in Yaa-DKO males (Fig. 2a; Supplementary Fig. 2A). Immunofluorescence staining confirmed these findings and revealed that GCs in Yaa-DKO 
a

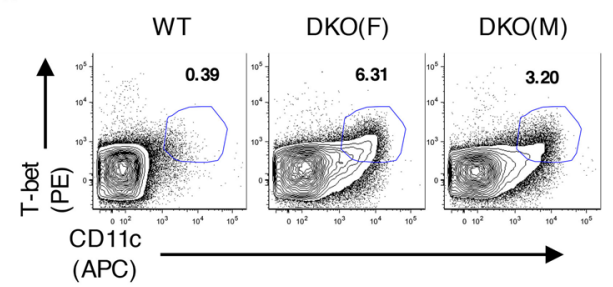

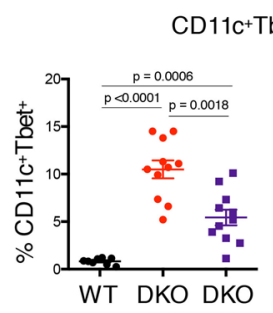

(F) (M)

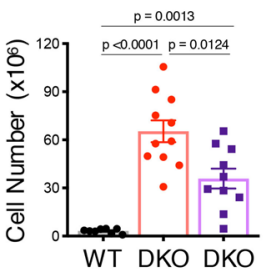

(F) (M)
C

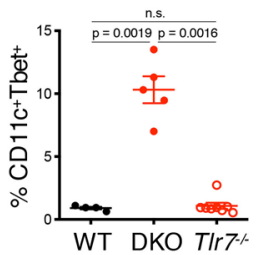

(F) $\mathrm{DKO}(\mathrm{F})$

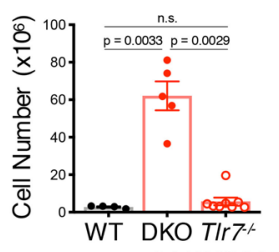

(F) $\mathrm{DKO}(\mathrm{F})$ d

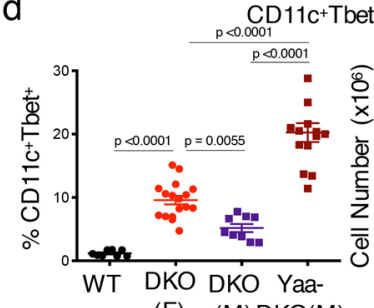

(F) (M) DK

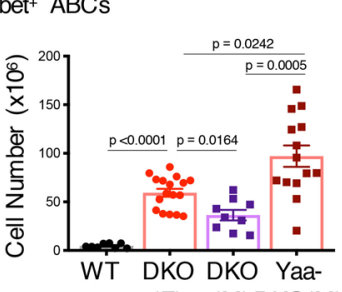

(F) (M) DKO(M)

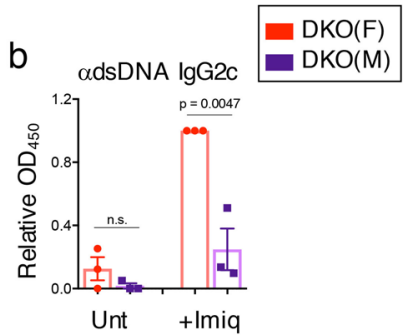

e

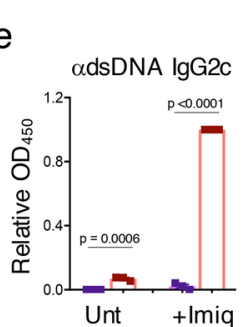

f

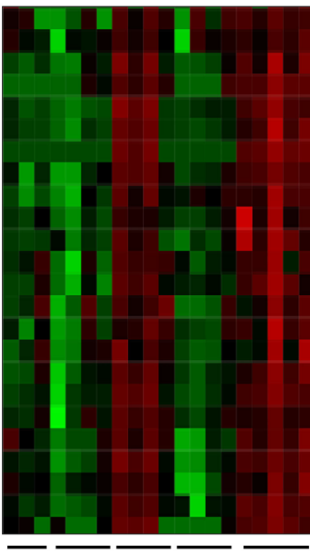

$\overline{\mathrm{WT}} \overline{\mathrm{WT}} \overline{\mathrm{DKO}} \overline{\mathrm{DKO}} \overline{\text { Yaa- }}$

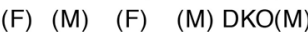

g adsDNA IgG2c

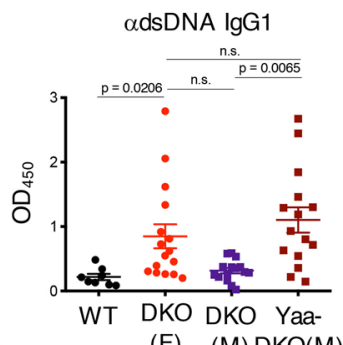

(F) (M) DKO(M)

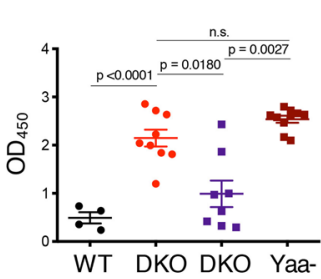

(F) (M) DKO(M) h

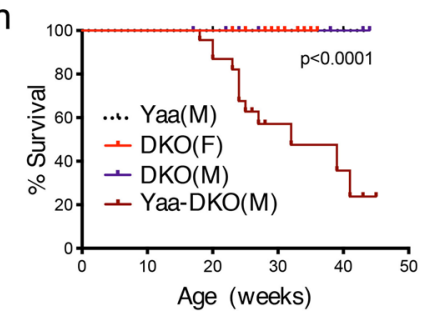

Fig. 1 TLR7 controls sex-specific differences in ABC formation and function. a Representative FACS plots and quantifications of CD11c ${ }^{+}$Tbet ${ }^{+} A B C s$ (gated on $\mathrm{B}^{2} 2 \mathrm{O}^{+}$) from spleens of aged (24+wk) female C57BL/6 (WT) (black circles), female DKO (F) (red circles), and male DKO (M) mice (purple squares). Data shows mean $\pm \mathrm{SEM} ; n=8$ for WT, $n=11$ for $\mathrm{DKO}(\mathrm{F}), n=10$ for DKO(M) over 8 independent experiments; $p$-value by Brown-Forsythe and Welch ANOVA followed by Games-Howell's test for multiple comparisons. b Pooled ELISA data for anti-dsDNA IgG2c antibodies from supernatants of sorted $\mathrm{ABCs}\left(\mathrm{B} 22 \mathrm{O}^{+} \mathrm{CD} 19+\mathrm{CD} 11 \mathrm{c}^{+} \mathrm{CD} 11 \mathrm{~b}^{+}\right)$from spleens of aged $(24+w k) \mathrm{DKO}(\mathrm{F})$ and $\mathrm{DKO}(\mathrm{M})$ mice after culturing with Imiquimod for $7 \mathrm{~d}$. Data shows mean $\pm \mathrm{SEM} ; n=3 ; p$-value by unpaired two-tailed $t$-tests. c Quantifications of CD11c ${ }^{+}$Tbet $^{+}$ABCs from the spleens of aged (24+wk) female WT, DKO(F), and female T/r7-deficient DKO (TIr7-/-.DKO(F)) mice (red open circles). Data show mean \pm SEM; $n=4$ for WT, $n=5$ for DKO(F), $n=8$ for T/r $7^{-/}$. DKO (F) mice over 4 independent experiments; $p$-value by Brown-Forsythe and Welch ANOVA followed by Games-Howell's test for multiple comparisons. d Quantifications of CD11c ${ }^{+}$Tbet $^{+}$ABCs (gated on B220 ${ }^{+}$) from the spleens of aged (24+wk) WT, DKO(F), DKO(M), and male Yaa-DKO(M) mice (maroon squares). Data shows mean $\pm \mathrm{SEM} ; n=8$ for WT, $n=17$ for DKO(F), $n=9$ for DKO(M), $n=14$ for Yaa-DKO(M) over 8 independent experiments; $p$-value by Brown-Forsythe and Welch ANOVA followed by Games-Howell's test for multiple comparisons. e Pooled ELISA data for antidsDNA IgG2c antibodies from supernatants of sorted ABCs from aged (24+wk) DKO(M) or Yaa-DKO(M) mice after culturing with Imiquimod for 7d. Data shows mean $\pm \mathrm{SEM} ; n=3 ; p$-value by unpaired two-tailed $t$-tests. $\mathbf{f}$ Autoantigen microarray showing the relative autoantibody levels in the serum of aged (24+wk) female WT (F), male WT (M), DKO(F), DKO(M), and Yaa-DKO(M) mice; $n=3$ for WT(F), $n=4$ for WT(M), DKO(F), and DKO(M), $n=5$ for Yaa-DKO(M). $\mathbf{g}$ Pooled ELISA data for anti-dsDNA IgG2c and IgG1 in the serum from the indicated aged (24+wk) mice. Data shows mean \pm SEM; for anti-dsDNA IgG2c, $n=4$ for WT, $n=9$ for DKO(F), $n=8$ for DKO(M), $n=10$ for Yaa-DKO(M); for anti-dsDNA IgG1, $n=8$ for WT, $n=16$ for DKO(F), $n=14$ for DKO(M); $n=15$ for Yaa-DKO(M); $p$-value by Brown-Forsythe and Welch ANOVA followed by Games-Howell's test for multiple comparisons. $\mathbf{h}$ Plot showing survival rate of Yaa control, DKO(F), DKO(M), and Yaa-DKO(M) mice across 45 weeks. Data represents a cohort of 14 Yaa control, 16 DKO $(F), 9 \mathrm{DKO}(\mathrm{M})$, and 31 Yaa-DKO(M) mice; $p$-value by Mantel-Cox test.

males were smaller and less well-organized than those in DKO females (Fig. 2b; Supplementary Fig. 2B). DKO females also demonstrated a greater expansion of $\mathrm{PB} / \mathrm{PCs}$ than $\mathrm{DKO}$ males (Fig. 2c). Tlr7 duplication in Yaa-DKO males reversed this effect and strongly promoted the accumulation of $\mathrm{PB} / \mathrm{PCs}$, which was primarily observed in the spleen but not in the BM (Fig. 2c; Supplementary Fig. 2C). No sex-based differences were detected in other B cell compartments (Supplementary Fig. 2D-G). Other parameters known to promote spontaneous GC responses such as the ratio between $\mathrm{T}_{\mathrm{FH}}$ and $\mathrm{T}_{\mathrm{FR}}$ or the dual production of IFN $\gamma$ 
a
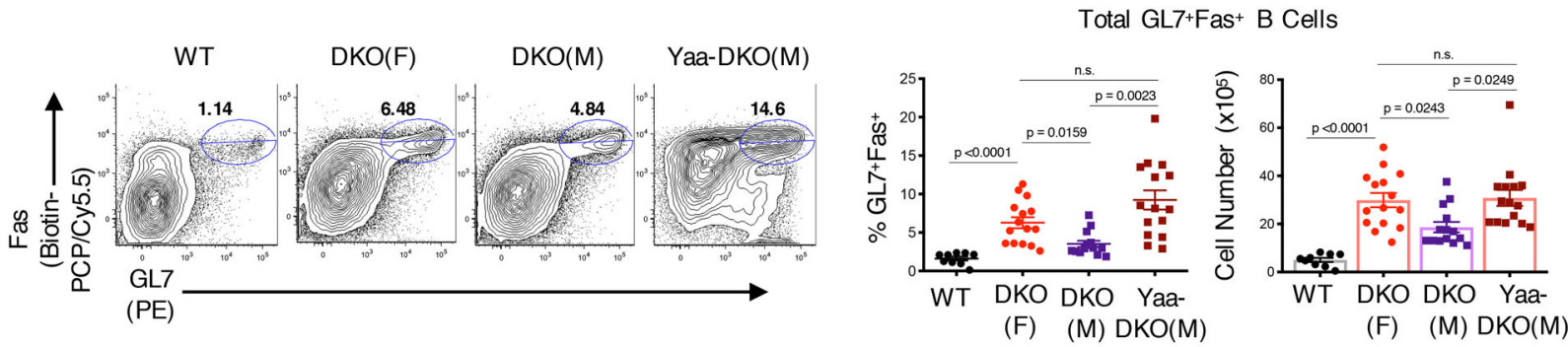

b
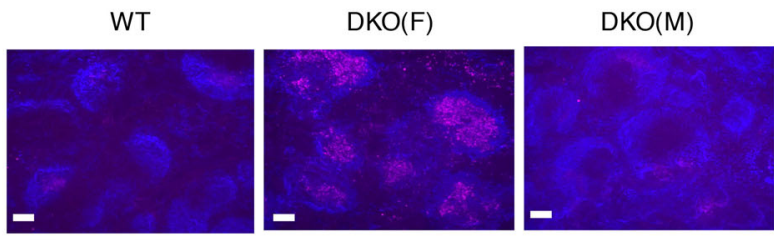

Yaa-DKO(M)

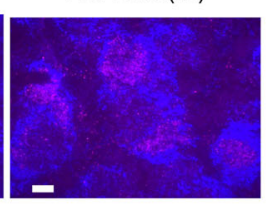

C

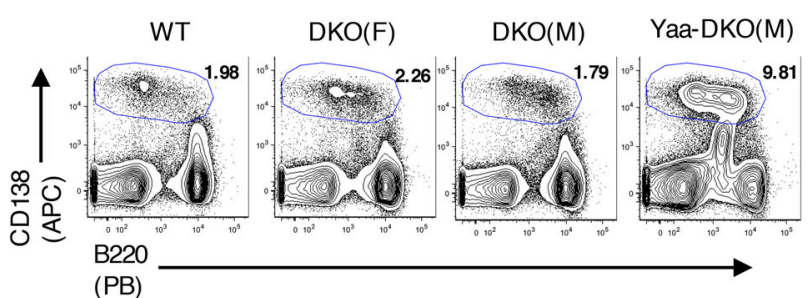

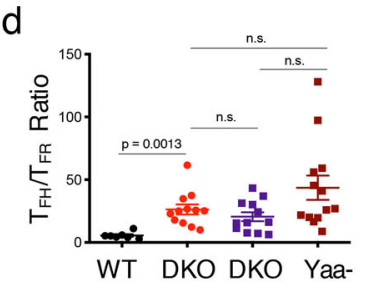

(F) (M) DKO(M)

e

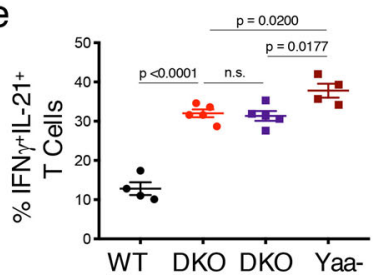

(F) (M) DKO(M)

f

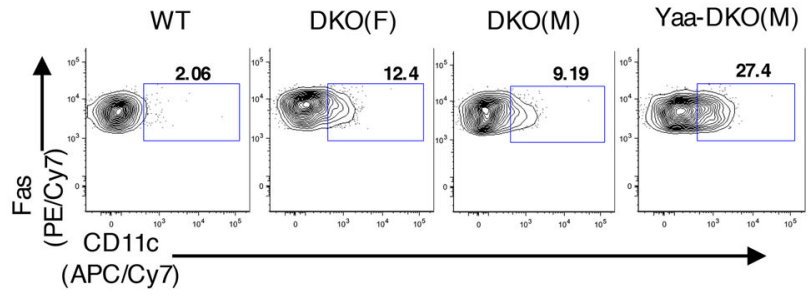

g

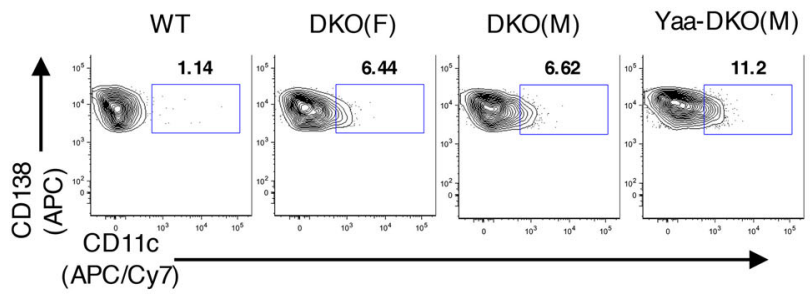

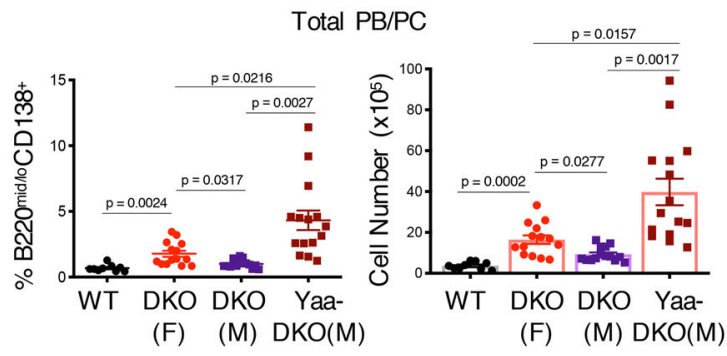

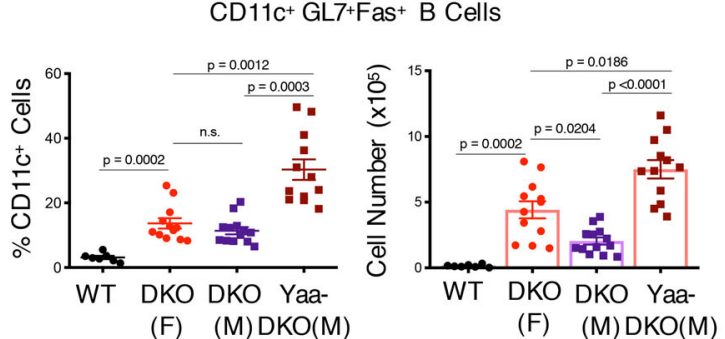

$\mathrm{CD} 11 \mathrm{c}^{+} \mathrm{PB} / \mathrm{PCs}$
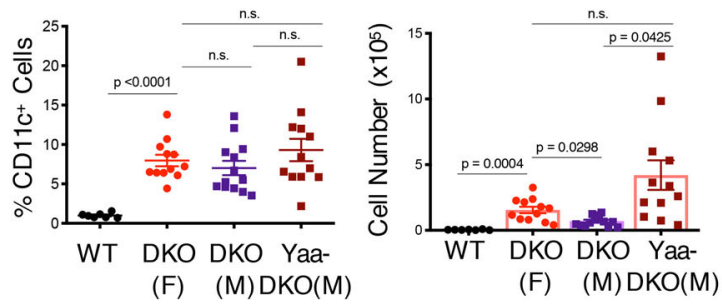

and IL-21 were comparable between DKO females and males and only minimally affected by Tlr7 duplication (Fig. 2d, e; Supplementary Fig. 2H-J). Thus, DKOs exhibit a sex-specific accumulation of GC B cells and PB/PCs, which can be regulated in a Tlr7dependent manner.
Given the sex-bias in the accumulation of ABCs as well as of GC B cells and PB/PCs, we next investigated whether these populations might be related. An analysis of the GC B cell population in DKO females revealed that a subset of these cells expressed CD11c and that the numbers of CD11c ${ }^{+}$GC B cells 
Fig. 2 Sex-dependent accumulation of GC B cells and PB/PCs in DKO mice. a Representative FACS plots and quantifications of total GC B cells (gated on

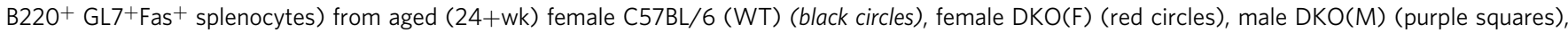
and male Yaa-DKO(M) mice (maroon squares). Data show mean \pm SEM; $n=9$ for WT, $n=15$ for DKO(F) and Yaa-DKO(M), $n=14$ for DKO(M) over 9 independent experiments; $p$-value by Brown-Forsythe and Welch ANOVA followed by Games-Howell's test for multiple comparisons. b Representative immunofluorescence images of $\mathrm{B}_{220^{+}}$(blue) $\mathrm{GL}^{+}$(pink) GCs in spleens from the indicated mice. Data representative of at least 4 frames for at least 2 mice per genotype. Bars show $50 \mu \mathrm{m}$. c Representative FACS plots and quantifications of total PB/PC (B220mid/lo $\left.\mathrm{CD} 138^{+}\right)$in spleens of the indicated mice. Data show mean $\pm \mathrm{SEM} ; n=9$ for $\mathrm{WT}, n=14$ for $\mathrm{DKO}(\mathrm{F})$ and $\mathrm{DKO}(\mathrm{M}), n=15$ for Yaa-DKO(M) over 9 independent experiments; $p$-value by Brown-Forsythe and Welch ANOVA followed by Games-Howell's test for multiple comparisons. d Quantification of the FACS plots showing $T_{F H}$ (CD4 ${ }^{+}$ $\left.\mathrm{PD}^{\text {hiCXCR5 }}{ }^{+} \mathrm{Foxp}^{-}\right)$to $\mathrm{T}_{\mathrm{FR}}\left(\mathrm{CD} 4^{+} \mathrm{PD} 1^{\text {hi }} \mathrm{CXCR} 5^{+}\right.$Foxp3 $\left.{ }^{+}\right)$ratio in spleens from the indicated mice. Data show mean $\pm \mathrm{SEM} ; n=7$ for $\mathrm{WT}, n=12$ for $\mathrm{DKO}(\mathrm{F})$ and $\mathrm{DKO}(\mathrm{M}), n=13$ for Yaa-DKO(M) over 7 independent experiments; $p$-value by Brown-Forsythe and Welch ANOVA followed by Games-Howell's test for multiple comparisons. e Quantification of FACS plots showing IFN $\gamma^{+} \mathrm{IL}-21^{+} \mathrm{T}$ cells in spleens of the indicated mice following $4 \mathrm{~h}$ treatment of splenocytes with PMA/lonomycin. Data show mean \pm SEM; $n=4$ for WT and Yaa-DKO(M), $n=5$ for DKO(F) and DKO(M) over 4 independent experiments; $p$-value by Brown-Forsythe and Welch ANOVA followed by Games-Howell's test for multiple comparisons. $\mathbf{f}, \mathbf{g}$ Representative

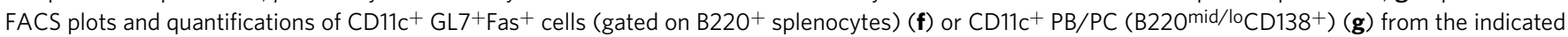
mice. Data show mean \pm SEM; $n=7$ for WT, $n=12$ for DKO(F) and Yaa-DKO(M), $n=3$ for DKO(M) over 7 independent experiments; $p$-value by Brown-Forsythe and Welch ANOVA followed by Games-Howell's test for multiple comparisons.

were significantly greater in DKO females than DKO males or WT controls (Fig. 2f; Supplementary Fig. 2K). CD11c ${ }^{+}$GC B cells were greatly increased in Yaa-DKO males (Fig. 2f). We also identified a population of $\mathrm{CD} 11 \mathrm{c}^{+} \mathrm{PB} / \mathrm{PCs}$ that accumulated in DKO females and, to an even greater extent, in Yaa-DKO males (Fig. 2g; Supplementary Fig. 2L). Thus, sex differences in lupus development in DKOs are accompanied by the aberrant accumulation of CD11c-expressing B cell effector subsets.

Oligoclonal expansion and interrelatedness of ABCs and $\mathrm{CD}_{11 c^{+}}$and $\mathrm{CD} 1 \mathrm{c}^{-} \mathrm{B}$ cell effector populations. To gain further insights into the effector B cell subsets that differentially expand in the spleens of DKO mice, we next compared their BCR repertoires. To evaluate the clonal landscape, we began by determining the contribution of the top 20 ranked clones to the overall repertoire by computing the D20 index ${ }^{34}$. We observed that the D20 index, or fraction of sequence copies contributing to the sum of the top 20 ranked clones, was lowest in follicular B cells (FoBs) and increased in ABCs, followed by GCB and finally being highest (most expanded) in the PB/PC pool (Fig. 3a). This order of large clone contribution by $\mathrm{B}$ cell subset was preserved in both DKO females and Yaa-DKO males (Supplementary Fig. 3A). Furthermore, when one studies the level of resampling of clones between replicate sequencing libraries as an independent measure of clone size, the same trend is preserved, with FoBs having the lowest degree of overlap and PB/PCs having the highest (Fig. 3b; Supplementary Fig. 3B). We next analyzed the level of somatic hypermutation (SHM), which revealed that $\mathrm{GCB}$ and $\mathrm{PB} / \mathrm{PC}$ fractions had the highest frequencies of clones with SHM, while the ABCs had a level of SHM that was intermediate between FoBs and GCB/PBs (Fig. 3c; Supplementary Fig. 3C, D). The SHM distribution trended by $\mathrm{B}$ cell subset rather than by mouse strain and the relative levels of SHM were preserved across these different B cell subsets irrespective of whether clones were unweighted or weighted by size (Fig. 3d; Supplementary Fig. 3C, D). Given the somewhat lower levels of SHM in the ABCs as compared to the other B cell populations, we next turned to the length of the third complementary determining region (CDR3) and heavy chain variable $(\mathrm{VH})$ gene usage as other general repertoire features. This analysis revealed longer CDR3 lengths for FoBs compared to the other B cell populations and $\mathrm{VH}$ gene usage that differed between FoBs and the other subsets (Fig. 3e, f). Taken together, this initial global repertoire analysis revealed that general repertoire features tended to map by B cell subset rather than by mouse strain, with FoBs having the greatest diversity, smallest clone size, and lowest level of SHM and GCB/PBs having the highest. ABCs instead tended to be intermediate with most of these measures.

To further assess the relationships of ABCs with the other $\mathrm{B}$ cell effector populations that aberrantly expand in DKO females and Yaa-DKO males, we sorted and analyzed the various populations from individual mice, stratifying the GCB and $\mathrm{PB} /$ PC subsets by CD11c expression. CD11c is differentially expressed on DN B cells in human lupus and we wondered if similar interconnections existed in the context of the DKO females and Yaa-DKO male models. In particular, CD11 $c^{+}$DN2 cells are transcriptionally and epigenetically poised to become $\mathrm{PBs}$ in human $\mathrm{SLE}^{35}$. If this also occurs in these models of murine SLE, then $\mathrm{CD}_{11 \mathrm{c}^{+}} \mathrm{ABCs}$, which are phenotypically and functionally overlapping with DN2, may exhibit a higher degree of clonal overlap with $\mathrm{PB} / \mathrm{PCs}$ than with GCBs or with $\mathrm{CD} 11 \mathrm{c}^{-}$ subsets. We therefore visualized overlapping clones from DKO female and Yaa-DKO male mice as strings and in Venn Diagrams (Fig. 3g, h; Supplementary Fig. 3E-J). These visualizations revealed a high level of clonal overlap across all subsets, including hundreds of clones that were present in all the subsets. To quantify and compare the level of overlap between the different subsets, we compared the Jaccard index, in which each clone is only counted once in each subset, and the Cosine similarity, in which clone size is also taken into account (Fig. 3i; Supplementary Fig. 3J, K). Neither measure revealed a consistent pattern of similarity with respect to $\mathrm{CD} 11 \mathrm{c}$ status. However, ABCs were consistently most highly associated with $\mathrm{PB} / \mathrm{PCs}$.

To further verify the relationships between ABCs and the other effector populations in DKOs, we crossed them with Tbet-zsGreenT2A-CreER ${ }^{\mathrm{T} 2}$-Rosa26-loxP-STOP-loxP-tdTomato mice (termed ZTCE-DKO), where cells expressing T-bet co-express zsGreen and can be traced with Tamoxifen-inducible tdTomato expression $^{36}$. These mice allow for the detection of stable T-betexpressing cells $\left(\mathrm{zsGreen}^{+} \mathrm{tdTomato}^{+}\right.$) and cells that previously but no longer express T-bet $\left(\text { zsGreen }^{-} \text {tdTomato }^{+}\right)^{36}$. ABCs expressed high levels of both ZsGreen and tdTomato, indicating stable expression of T-bet (Fig. 3j, k). CD11c ${ }^{+}$and CD11c ${ }^{-}$GC B cells contained both $\mathrm{zsGreen}^{+}$tdTomato $^{+}$and $\mathrm{zsGreen}^{-}$tdTomato $^{+}$ although the $\mathrm{CD}_{11 \mathrm{c}^{-}}$subset was preferentially $\mathrm{zsGreen}^{-} \mathrm{tdTo}^{-}$ mato $^{+}$suggesting that both populations can originate from $\mathrm{T}$-bet expressing cells but that $\mathrm{CD} 11 \mathrm{c}^{+} \mathrm{GC} \mathrm{B}$ cells include a greater fraction of stable T-bet expressors (Fig. 3j, k). Although no longer expressing ZsGreen, a substantial fraction of CD11c ${ }^{+} \mathrm{PB} / \mathrm{PCs}$ and $\mathrm{CD}_{11 \mathrm{c}^{-}} \mathrm{PB} / \mathrm{PCs}$ were tdTomato $^{+}$, suggesting that both these populations can derive from T-bet-expressing B cells (Fig. 3j, k). In combination with our clonal overlap analyses, these findings suggest that, in this autoimmune setting, $\mathrm{ABCs}$ share lineage 


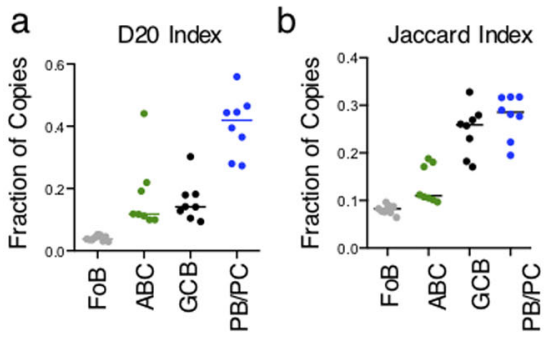

e
C

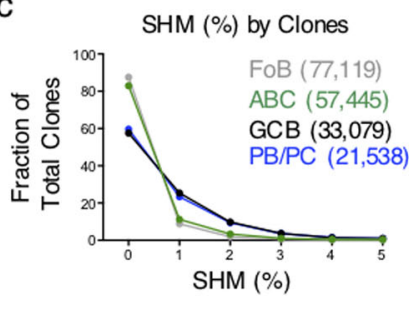

f d

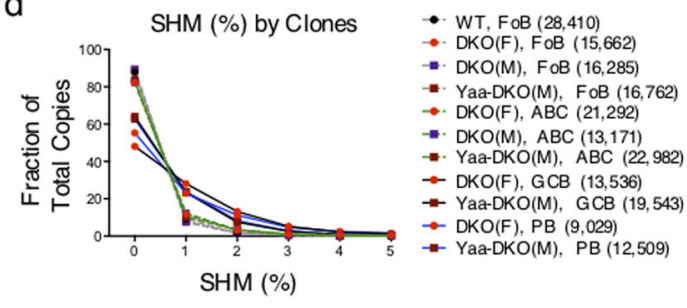

Top $20 V_{H}$ Genes
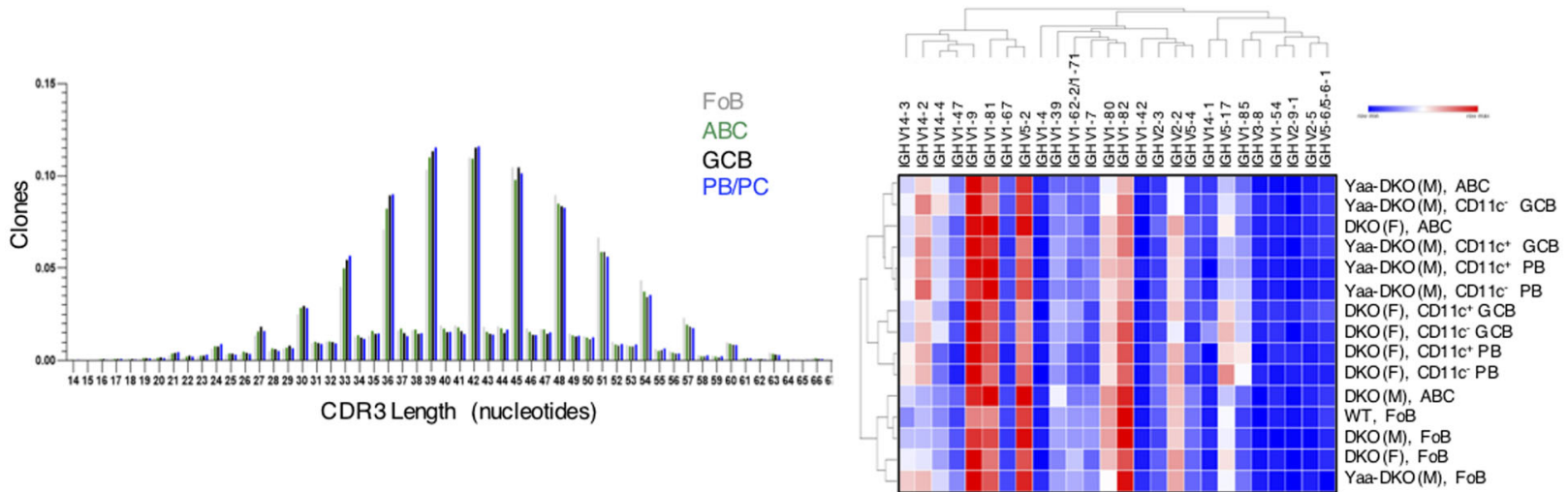

g

h

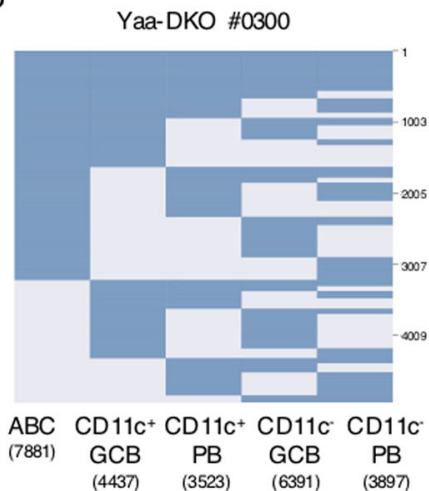

Yaa-DKO \#0300
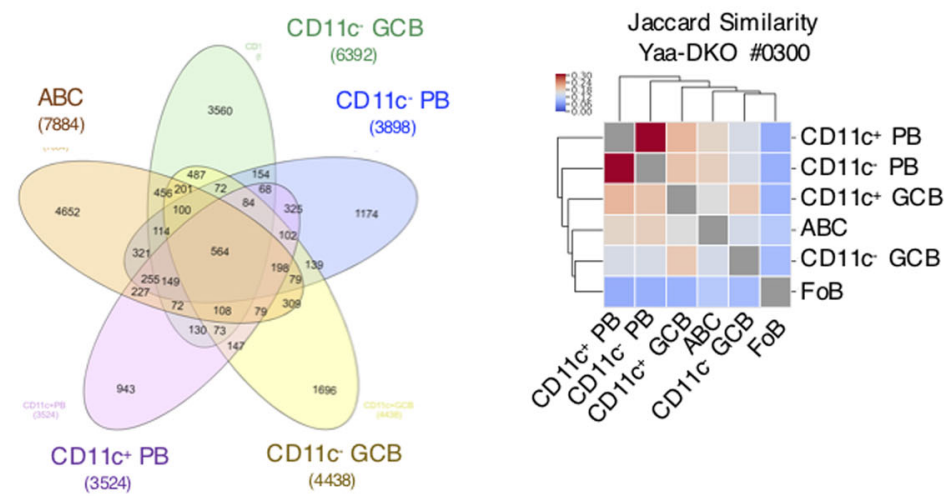

j

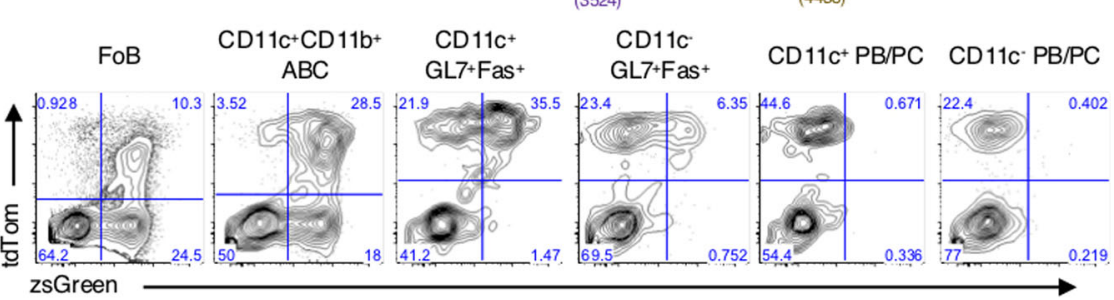

$\mathrm{k}$
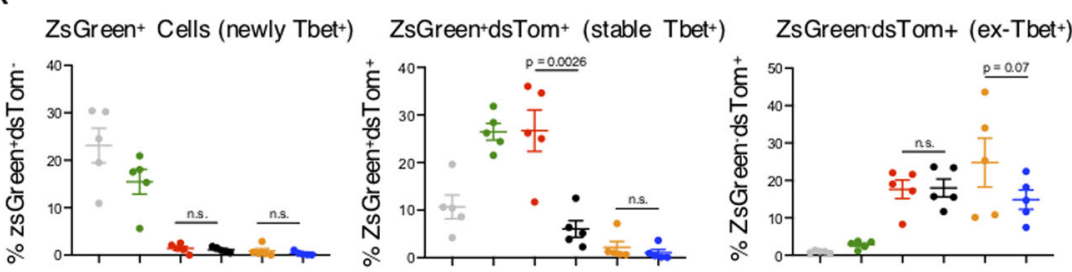

$\mathrm{FoB}$

$\mathrm{ABC}$

$\mathrm{CD} 11 \mathrm{c}^{+} \mathrm{GL} 7+\mathrm{Fas}^{+}$

CD11C' GL7+Fas

$\mathrm{CD} 11 \mathrm{C}^{+} \mathrm{PB} / \mathrm{PC}$

$\mathrm{CD} 11 \cdot \mathrm{PB} / \mathrm{PC}$

relationships with both $\mathrm{CD} 11 \mathrm{c}^{+}$and $\mathrm{CD} 11 \mathrm{c}^{-} \mathrm{GC} \mathrm{B}$ cell and $\mathrm{PB} / \mathrm{PC}$ populations.

Enrichment for an ISG signature differentiates ABCs from DKO females and males. While ABCs from female and male DKOs exhibited similar BCR repertoire features, their marked differences in function and differentiation suggested that they might employ distinct molecular programs. To gain insights into these mechanisms, we compared their transcriptome by RNA-seq (Fig. 4a). Gene set enrichment (GSEA) and CPDB pathway analyses revealed that $\mathrm{ABCs}$ from $\mathrm{DKO}$ females were enriched for pathways related to SLE pathogenesis, interferon (IFN) responses, and TLR and complement cascades (Fig. 4b; Supplementary Fig. 
Fig. 3 B cell effector populations exhibit a high degree of interrelatedness. IgH sequencing was performed on sorted FoBs $\left(B 220^{+} C D 11 c^{-} C D 11 b^{-} C D 23^{+}\right)$

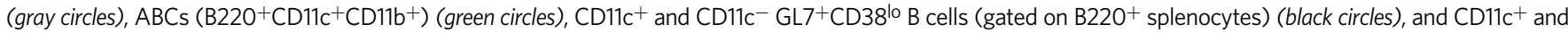
CD11 ${ }^{-}$PB/PCs (B220lo CD138+) (blue circles) from aged (24+wk) female C57BL/6 (WT), female DKO(F), male DKO(M), and male Yaa-DKO(M) mice. a D20 index of each population aggregated across all strains. D2O indicates the number of total copies in the top 20 clones as a fraction of total copies across all clones. Data show mean with each dot representing a single mouse; $n=8$ mice. $\mathbf{b}$ Plot showing the level of overlap between clones in different sequencing libraries prepared from the same sample aggregated across all strains. For each comparison, each clone was only counted once (no weighting for clone size) and functional overlap was computed using the Jaccard Index. Data show mean with each dot representing a single mouse; $n=8$ mice. c Plot showing somatic hypermutation (SHM) as a fraction of total clones for each subset aggregated across mouse strains. The numbers in parenthesis indicate the total number of clones in the given subset. Each clone counts once for each subset. If a clone overlaps in multiple subsets, the SHM for each subset was calculated just for the sequences in a given subset. $\mathbf{d}$ The SHM by clones is shown across each mouse strain/subset combination as in Fig. 3c. e Plot showing CDR3 length (in nucleotides) of clones aggregated across all strains. Each clone is counted once in each subset/strain combination; $n=8$. $\mathbf{f}$ Heatmap showing usage of the 20 most frequent $\mathrm{VH}$ genes. Each clone is counted only once. Data are normalized by row and visualized in Morpheus using the default settings. $\mathbf{g}$ Plot showing clones (rows) that overlap between at least two B cell subsets (columns). Numbers along the right side of the plot indicate clone counts. Data from a single analysis of subsets from a Yaa-DKO mouse. $\mathbf{h}$ Venn diagram showing clonal overlap where numbers indicate clone counts in the different subset interactions. Data from a single analysis of subsets from a Yaa-DKO mouse. i Heatmap showing Jaccard similarity (fraction of clones that overlap between different twosubset comparisons). Diagonal values are excluded for scaling. Data from a single analysis of subsets from a Yaa-DKO mouse. $\mathbf{j}, \mathbf{k}$ Female ZTCE-DKO mice were treated with tamoxifen to mark Tbet-expressing cells with tdTomato expression for 3d. Representative FACS plots (j) and quantifications (k) of zsGreen and tdTomato expression from the indicated populations. Data show mean $\pm S E M ; n=5$ over 2 independent experiments; $p$-value by paired two-tailed $t$-tests.

4A-C). In line with the known upregulation of both Type I and Type II IFN signatures in SLE patients, female ABCs were enriched for IFNa and IFN $\gamma$ responses and upregulated several IFN stimulated genes (ISGs) expressed in SLE PBMCs (Fig. 4b; Supplementary Fig. 4B) ${ }^{37}$. Male ABCs were instead enriched for pathways related to Rho GTPase signaling and platelet activation (Fig. 4c; Supplementary Fig. 4D, E). Given the profound effects of Tlr7 duplication on the ABCs of DKO males, we next sorted $\mathrm{ABCs}$ from Yaa-DKO males and compared their transcriptome to that of $\mathrm{ABCs}$ from DKO males (Fig. 4d). Similar to what was observed in female ABCs, the top pathways upregulated in ABCs from Yaa-DKO males were those related to IFN responses (Fig. 4e; Supplementary Fig. 4F, G). ABCs from DKO males were instead enriched for genesets related to hemostasis and platelet activation (Supplementary Fig. 4H, I). Thus, enrichment for an ISG signature differentiates $\mathrm{ABC}$ from $\mathrm{DKO}$ females and males and Tlr7 duplication promotes the upregulation of ISGs in male ABCs.

We next employed ATAC-seq to investigate the chromatin landscape of ABCs derived from the different DKOs. We identified at least 85,000 peaks in ABCs from female, male, and Yaa-DKO male mice (Supplementary Fig. 4J; Supplementary Table 1; Supplementary Data 1-2). Tlr7 overexpression induced sufficient changes in the chromatin landscape to enable a motif analysis of the differentially accessible regions (DAR) of YaaDKO male ABCs versus male ABCs. Peaks upregulated in ABCs from Yaa-DKO males were enriched for motifs known to be bound by IRFs and NFKB family members (Fig. 4f). Peaks upregulated in $\mathrm{ABCs}$ from $\mathrm{DKO}$ males were instead enriched for ETS binding sites (Supplementary Fig. 4K). Consistent with the transcriptional profiles and the enrichment in IRF binding motifs, loci that were differentially accessible in ABCs from Yaa-DKO males as compared to DKO males included a number of ISGs like Cxcl11, Ifi44, and Ifitm3 (Supplementary Fig. 4L). Thus, differences in ISG expression by ABCs are accompanied by a differential enrichment for IRF binding motifs.

We hypothesized that the aberrant gain of an IFN signature by $\mathrm{ABCs}$ in females was linked to intrinsic alterations in the epigenetic signatures of these cells and was not simply due to exposure to an IFN-rich environment. To address this hypothesis, we employed the CUT\&RUN technique to compare the global loss of a repressive chromatin mark, $\mathrm{H} 3 \mathrm{~K} 27 \mathrm{me} 3$, at key regulatory loci in $\mathrm{ABC}$ and $\mathrm{FoB}$ populations sorted from the same mice. Principal component analysis (PCA) of $\mathrm{H} 3 \mathrm{~K} 27 \mathrm{me} 3$ peaks in $\mathrm{ABCs}$ and FOBs from DKO females and males resulted in distinct clustering of samples based on sex and B cell subsets (Supplementary Fig. 4M). Genome-wide comparison of ABCs and FOBs from DKO females showed distinct loss of H3K27me3 signals at several loci among which Tbx21, the classical ABC marker, showed strongest loss in F-ABCs (Fig. 4g; Supplementary Fig. 4N-P). ABCs from DKO males demonstrated a similar loss of $\mathrm{H} 3 \mathrm{~K} 27 \mathrm{me} 3$ signals at $\mathrm{Tb} \times 21$ when compared to FOBs (Fig. 4g). Notably, however, only ABCs from females but not those from males showed selective loss of H3K27me3 marks at ISGs like the CXCL cluster (Fig. 4h; Supplementary Data 3) suggesting that ABCs in DKO females have intrinsic alterations in their epigenetic signatures that can predispose them to aberrantly gain an IFN signature.

Given that loss of $\mathrm{H} 3 \mathrm{~K} 27 \mathrm{me} 3$ signals has been shown to accompany XCI, we next specifically investigated the H3K27me3 peaks on the X-chromosome of $\mathrm{ABCs}$ and $\mathrm{FOBs}$ from $\mathrm{DKO}$ females. To gain insights into distinct regulation at various loci on the X-chromosome, we plotted Wald's z-statistic (X-chromosome deactivation score) obtained from DEseq2 analysis on the 3092 H3K27me3 peaks on the X-chromosomes between ABCs and FoBs from DKO females. H3K27me3 X-chromosome deactivation score plot showed distinct loss of H3K27me3 at over 2092 loci in ABCs as compared to FoBs that also included regulatory loci surrounding the Tlr7 gene (Fig. 4i). A closer look at the Tlr7 locus showed that there was a total of 3 peaks (chrX:167770493167776995, chrX:167827673-167830728 and chrX:168121697168125788) within the $T l r 7$ neighborhood that were deactivated (Fig. 4i). The deactivated loci were located far away from the Tlr7 gene body but remained in the same topologically associated domains (TADs) suggesting selective deactivation of repressive H3K27me3 marks within the intra-TAD boundaries of the Tlr7 gene locus. These results suggest that selective loss of repressive chromatin marks in ABCs of DKO females can contribute to the increased expression of X-chromosome linked genes like Tlr7.

Aberrant expansion of CD11c-expressing pre-GC B cells and PBs in DKO females. In addition to ABCs, populations of CD11c-expressing GC B cells and PB/PCs also accumulate to a greater extent in DKO females than DKO males suggesting that they also contribute to the sex-bias in disease development. Since the phenotypic and molecular characteristics of these populations are largely unknown, we investigated them in greater detail. $\mathrm{CD} 11 \mathrm{c}^{+} \mathrm{GC} \mathrm{B}$ cells shared several phenotypic features with $\mathrm{ABCs}$ including high expression of T-bet, Fcrl5, and Cxcr3 (Supplementary Fig. 5A). As compared to CD11 $\mathrm{c}^{-}$ 

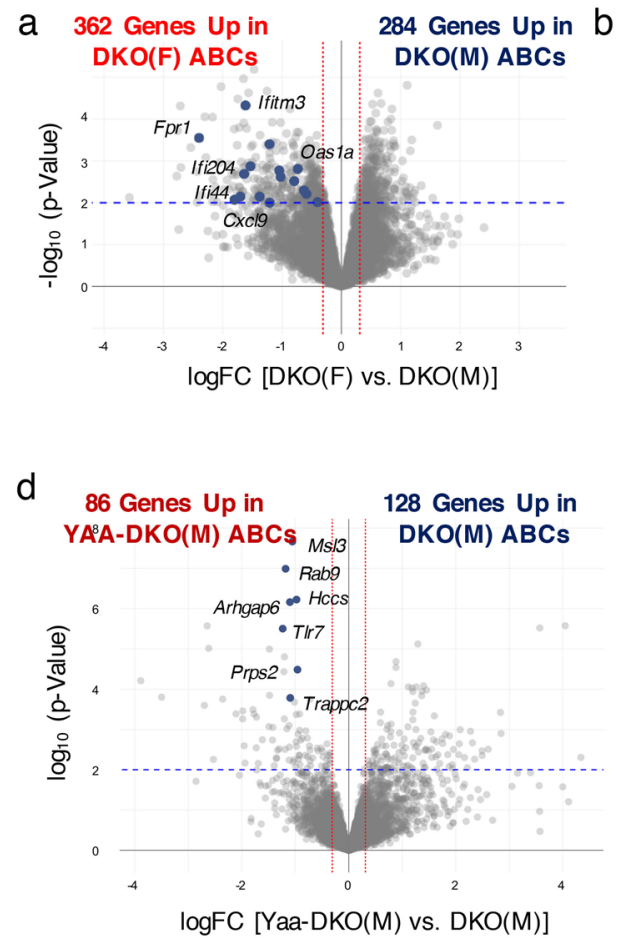

g

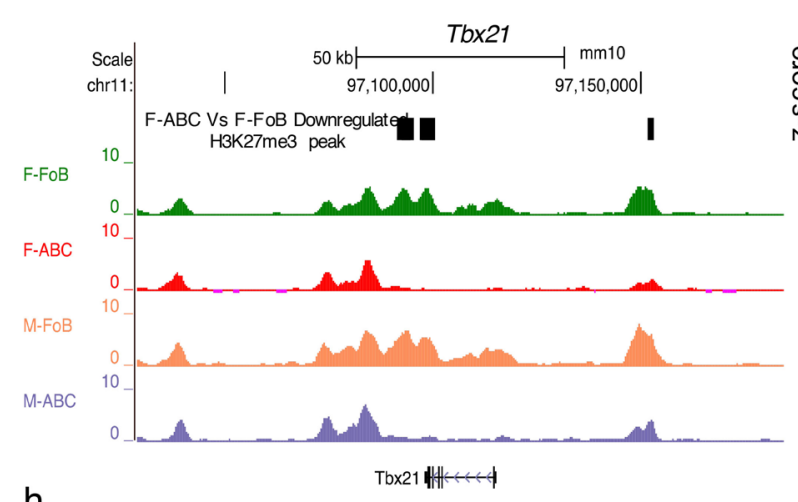

h

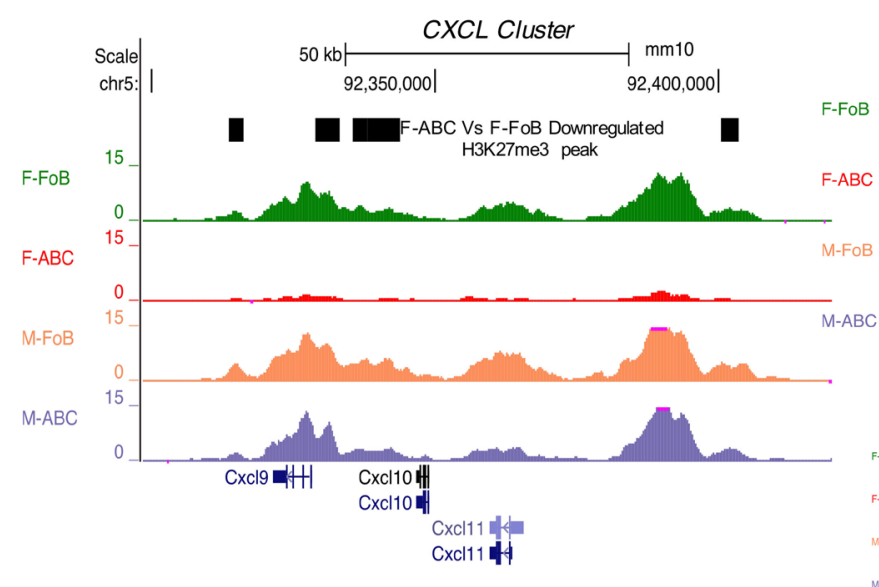

C

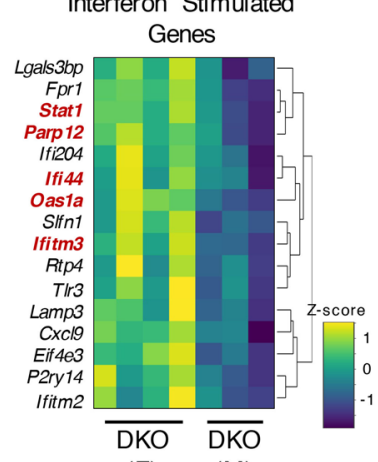

e
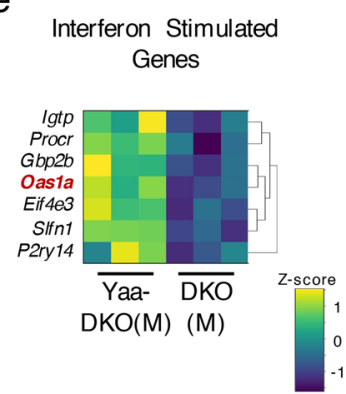

RhoGTPase Signaling

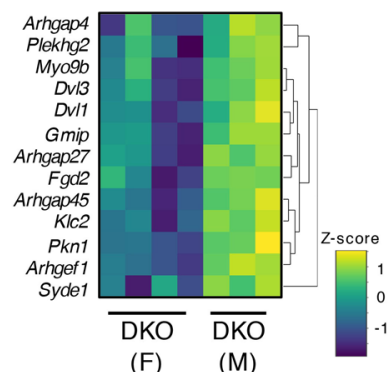

(F) f

\begin{tabular}{|c|c|c|}
\hline \multicolumn{3}{|c|}{$\begin{array}{c}\text { Upregulated Peaks in } \\
\text { Yaa-DKO(M) vs. DKO(M) ABCs }\end{array}$} \\
\hline Motif & $P$ value & Best Match \\
\hline$=11$ & $10^{-163}$ & IRF \\
\hline & $10^{-123}$ & Rel_class \\
\hline $\mathrm{ATG}$ & $10^{-52}$ & Pou2F2 \\
\hline CACCTG & $10^{-40}$ & $\mathrm{~b} H L \mathrm{H}(\mathrm{E} 2 \mathrm{~A})$ \\
\hline $\mathrm{T}_{\triangle A}=I \mathrm{C} A$ & $10^{-36}$ & bZIP(BATF) \\
\hline
\end{tabular}
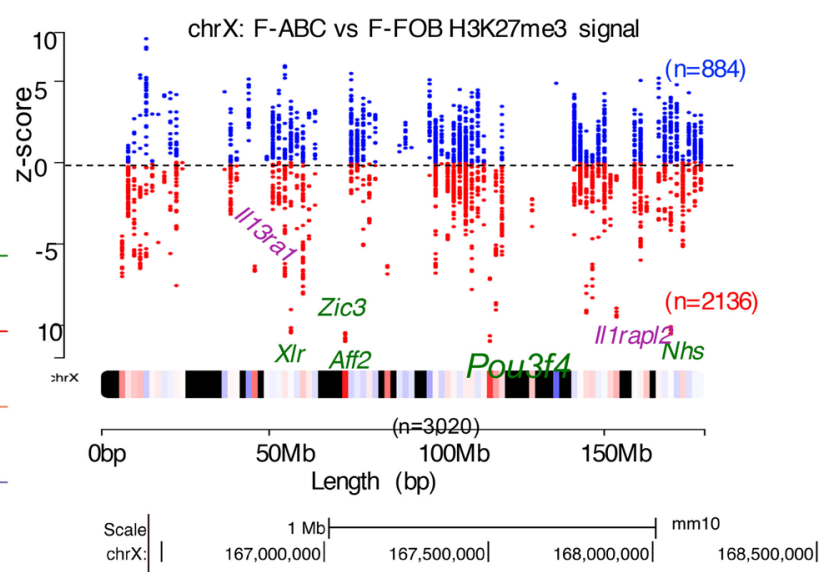

TAD

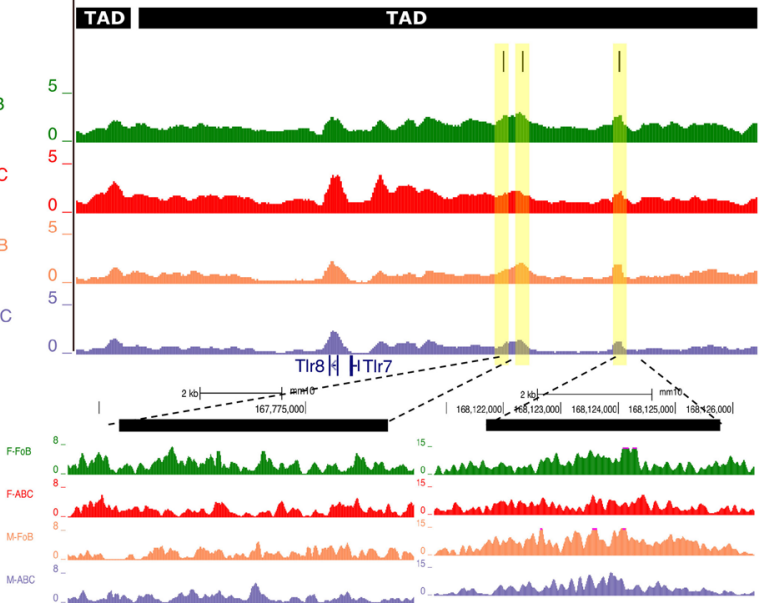

GC B cells, moreover, the transcriptome of sorted $\mathrm{CD} 11 \mathrm{c}^{+} \mathrm{GC} B$ cells was enriched for $A B C$ genesets and for IFN responses (Fig. 5a, b; Supplementary Fig. 5B-C). Despite expressing comparable transcript levels of several classical GC target genes, including Bcl6, Irf8, and Spib, the levels of BCL6 protein were lower in the $\mathrm{CD} 11 \mathrm{c}^{+}$than in the $\mathrm{CD} 11 \mathrm{c}^{-}$populations (Fig. 5c;
Supplementary Fig. 5D). In line with the notion that intermediate expression of BCL6 protein in B cells has been associated with a pre-GC B cell state ${ }^{38}$, the $\mathrm{CD} 11 \mathrm{c}^{+}$population contained increased frequencies of BCL6 ${ }^{\text {mid }} \mathrm{IRF}^{+}{ }^{+} \mathrm{B}$ cells, a profile associated with pre-GC B cells, and was enriched in a pre-GC B cell signature by GSEA (Fig. 5d, e). BCR signaling, as 
Fig. 4 DKO ABCs upregulate ISGs in a sex-specific manner. a-e RNA-seq analyses were performed on sorted $A B C s\left(B 220^{+} C D 19+C D 11 c^{+} C D 11 b^{+}\right)$from aged (24+wk) female DKO (F), male DKO (M), and male Yaa-DKO (M) mice. a Volcano plot showing differentially expressed genes ( $p<0.01$ after BenhaminiHochberg false discovery rate (FDR) was used to correct for multiple comparisons) in ABCs from DKO(F) and DKO(M) mice. Genes in blue show Interferon Stimulated Genes (ISGs) represented in Fig. 4b. b Heatmap showing the differential expression of ISGs in ABCs from DKO(F) and DKO(M) mice. c Heatmap showing differentially expressed genes in the REACTOME_RhoGTPase_CYCLE geneset. $\mathbf{d}$ Volcano plot showing differentially expressed genes ( $p<0.01$ after Benhamini-Hochberg false discovery rate (FDR) was used to correct for multiple comparisons) in ABCs from Yaa-DKO(M) mice and DKO(M) mice. Genes in blue show genes on the Yaa translocation. e Heatmap showing the differential expression of ISGs in ABCs from Yaa-DKO(M) and DKO(M) mice. $f$ ATAC-seq was performed on $A B C$ s from aged (24+wk) DKO(F), DKO(M), and Yaa-DKO(M) mice. Motif enrichment analysis in ATAC-seq peaks that are significantly upregulated (logFC >1.5; EdgeR, QLF FDR <0.05) in ABCs from Yaa-DKO(M) as compared to DKO(M) mice. g-i CUT\&RUN for H3K27me3 marks was performed on ABCs from aged (24+wk) DKO(F) and DKO(M) mice. $\mathbf{g}$, h Representative UCSC genome browser tracks of H3K27me3 marks for Tbx21 gene (g) and CXCL gene cluster (h; CxCl9, CxCl10 \& CxCl11). The black boxes in each of the browser track represents genome co-ordinates of significantly downregulated H3K27me3 peaks from DKO(F) ABCs as compared to DKO(F) FoBs (2-fold down and $=<0.05$ FDR). i X-chromosome map of $3092 \mathrm{H3K} 27 \mathrm{me} 3$ peaks across the length the chromosome where each dot represents a H3K27me3 peak. Black bands on X-chromosome are devoid of H3K27me3 signal and the color spectrum represents various $\mathrm{H} 3 \mathrm{~K} 27 \mathrm{me} 3$ peaks. The $z$-score (X-chromosome inactivation score) is a DESeq2 Wald statistic showing gain or loss of H3K27me3 signal across 3092 peaks. A total of 2135 peaks had negative z-scores (downregulated) in DKO(F) ABCs as compared to DKO(F) FoBs and 884 had positive z-scores (upregulated). Genome browser track of H3K27me3 signals over the T/r7 locus, highlighted in yellow are the downregulated H3K27me3 peaks in DKO(F) ABCs. The black boxes on the top represent TAD boundaries on the X-chromosome called by machine-learning program Peakachu ${ }^{88}$ obtained from a murine $\mathrm{B}$ cell lymphoma $\mathrm{CH} 12^{89}$.

monitored by the phosphorylation of SYK and LYN, was furthermore significantly higher in $\mathrm{CD} 11 \mathrm{c}^{+}$than $\mathrm{CD} 11 \mathrm{c}^{-} \mathrm{GL}^{+}$ $\mathrm{Fas}^{+} \mathrm{B}$ cells (Fig. 5f). The CD11 $\mathrm{c}^{+}$subset furthermore was less proliferative than $\mathrm{CD}_{11 \mathrm{c}^{-}} \mathrm{GL7}^{+} \mathrm{Fas}^{+} \mathrm{B}$ cells (Fig. 5g, h). In contrast, the $\mathrm{CD} 11 \mathrm{c}^{+}$population upregulated pathways related to migration and apoptotic cell clearance and expressed high levels of MerTK, a critical efferocytic receptor (Fig. 5i, j). A greater percentage of CD11c $\mathrm{c}^{+} \mathrm{GL}^{+} \mathrm{Fas}^{+} \mathrm{B}$ cells than $\mathrm{CD} 11 \mathrm{c}^{-}$ $\mathrm{GL}^{+}{ }^{+} \mathrm{Fas}^{+} \mathrm{B}$ cells furthermore could engulf apoptotic thymocytes and this was coupled with upregulation of surface MHC-II expression (Supplementary Fig. 5E, F). Thus, the CD11c ${ }^{+}$GL7 ${ }^{+}$Fas $^{+} \mathrm{B}$ cells that aberrantly expand in DKO females likely represent $\mathrm{ABCs}$ that have acquired a pre-GC $\mathrm{B}$ cell phenotype and can both engulf and present apoptotic debris, thus potentially augmenting autoreactive responses.

To gain insights into the molecular profiles of the $\mathrm{CD} 11 \mathrm{c}^{+} \mathrm{PB} /$ PCs that also aberrantly expand in DKO females, we sorted $\mathrm{CD}_{11 \mathrm{c}^{+}}$and $\mathrm{CD} 11 \mathrm{c}^{-} \mathrm{PB} / \mathrm{PCs}$ from DKO females and compared their transcriptome by RNA-seq (Fig. 6a). Consistent with their expression of $\mathrm{CD} 11 \mathrm{c}, \mathrm{CD} 11 \mathrm{c}^{+} \mathrm{PB} / \mathrm{PCs}$ were enriched for $\mathrm{ABC}$ signatures and IFN responses (Fig. 6b; Supplementary Fig. 6A-C).

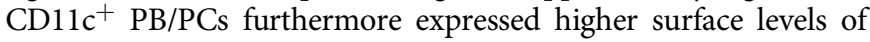
$\mathrm{ABC}$ markers like $\mathrm{Cxcr3}$ and Fcrl5 than $\mathrm{CD} 11 \mathrm{c}^{-} \mathrm{PB} / \mathrm{PCs}$, although, consistent with the fate mapping studies, they expressed only low levels of T-bet (Fig. 6c). Thus, $\mathrm{CD} 11 \mathrm{c}^{+} \mathrm{PB} / \mathrm{PCs}$ share several transcriptional and phenotypic similarities with $\mathrm{ABCs}$ despite downregulating T-bet expression. $\mathrm{CD}_{11 \mathrm{c}^{+}}$and $\mathrm{CD} 11 \mathrm{c}^{-}$ $\mathrm{PB} / \mathrm{PCs}$ expressed similar transcript levels of Prmd1 and Irf4 although the transcriptional programs normally regulated by Blimp1 and Irf4 in PB/PCs were enriched to a greater extent in $\mathrm{CD} 11 c^{-} \mathrm{PB} / \mathrm{PCs}$ than in $\mathrm{CD} 11 \mathrm{c}^{+} \mathrm{PB} / \mathrm{PCs}$ (Fig. 6d; Supplementary Fig. $6 \mathrm{D})$. $\mathrm{CD} 11 \mathrm{c}^{+} \mathrm{PB} / \mathrm{PCs}$ were more proliferative than $\mathrm{CD} 11 \mathrm{c}^{-}$ $\mathrm{PB} / \mathrm{PCs}$ and expressed higher levels of B220, MHC-II, and Ciita (Fig. 6e, f). As compared to $\mathrm{CD} 11 \mathrm{c}^{-} \mathrm{PB} / \mathrm{PCs}, \mathrm{CD} 11 \mathrm{c}^{+} \mathrm{PB} / \mathrm{PCs}$ upregulated pathways related to migration including chemokine receptors like $\mathrm{C} c r 3$ as well as the expression of pro-inflammatory cytokines such as Tnf and Illb and of inflammasome components like Nlrp3 (Fig. 6h-j). Taken together these data thus suggest that $\mathrm{CD} 11 \mathrm{c}^{+} \mathrm{PB} / \mathrm{PCs}$ represent a population of $\mathrm{PBs}$ with distinctive migratory and pro-inflammatory characteristics.

IRF8 and IRF5 cooperate in promoting the generation and differentiation of ABCs in DKO females. The finding that a substantial proportion of effector B cell populations were related to ABCs and previously expressed T-bet prompted us to examine the role of T-bet in the accumulation of ABCs and their progeny in DKO females. We thus generated CD23-Cre $T b x 21^{\text {flox/flox. }}$. DKO mice to specifically delete T-bet in B cells from DKO mice. Despite successful T-bet deletion, lack of B-cell T-bet did not significantly decrease the formation of $\mathrm{ABCs}$ as assessed by staining with $\mathrm{CD} 11 \mathrm{c}$ and $\mathrm{CD} 11 \mathrm{~b}$ and other $\mathrm{ABC}$ markers such as Fcrl5 and Cxcr3 (Fig. 7a-c). Lack of B cell T-bet also did not affect the accumulation of total or $\mathrm{CD} 11 \mathrm{c}^{+} \mathrm{GL}^{+} \mathrm{Fas}^{+} \mathrm{B}$ cells and $\mathrm{PB} / \mathrm{PCs}$ or the $\mathrm{T}_{\mathrm{FH}} / \mathrm{T}_{\mathrm{FR}}$ ratio (Fig. $7 \mathrm{~d}-\mathrm{h}$ ). Lack of $\mathrm{B}$ cell T-bet did, however, result in a profound decrease in anti-dsDNA IgG2c without a corresponding increase in anti-dsDNA IgG1 (Fig. 7i). Thus, in autoimmune-prone DKO females, B cell T-bet is not necessary for $\mathrm{ABC}$ generation or differentiation but is specifically required for the production of IgG2c autoantibodies.

Given that global IRF5 deletion profoundly decreased ABC formation in DKO females ${ }^{20,39}$, we next employed a similar strategy to investigate whether B-cell expression of IRF5 was specifically required for $\mathrm{ABC}$ generation and differentiation. Since IRF8 was also identified as a potential upstream regulator of $A B C s$ in DKO females versus males (Supplementary Fig. 7A), we also extended this analysis to B cell IRF8. ABC accumulation was significantly decreased in CD23-Cre.Irf5flox/+ $\mathrm{DKO}, \mathrm{CD} 23-\mathrm{Cre}$. Irf $5^{f l o x} / f^{\prime l o x} \mathrm{DKO}$, and CD23-Cre.Irf flox/flox DKO females (Fig. 8a, b). Lack of B-cell IRF5 and IRF8 also markedly affected the expansion of GC B cells and CD11 $\mathrm{c}^{+} \mathrm{PB} / \mathrm{PCs}$ but only the absence of IRF5 affected the accumulation of total PB/PCs (Fig. 8c-f). Absence of $B$ cell IRF5 or IRF8 also significantly impacted $T_{\mathrm{FH}}$ responses and the production of anti-dsDNA IgG2c antibodies (Fig. 8g; Supplementary Fig. 7B, C). To delineate the relative contributions of IRF5 and IRF8 to the generation of ABCs, we utilized an in vitro culture system ${ }^{20}$. Lack of either IRF5 or IRF8 reduced the ability of DKO B cells to differentiate into $\mathrm{ABCs}$ albeit not in an identical manner, since deletion of IRF8 impaired CD11c upregulation while lack of IRF5 diminished T-bet induction (Fig. 8h, i). Both IRF5 and IRF8 were required for the enhanced production of CXCL10 by DKO ABCs and ChIP-qPCR demonstrated increased binding of both IRF5, as we previously reported $^{20}$, and IRF8 to the regulatory regions controlling the Cxcl cluster of genes in DKO B cells (Fig. 8j, k). Deleting IRF8 in DKO B cells impaired the ability of IRF5 to bind to these regions and lack of IRF5 decreased binding of IRF8 to these sites (Fig. 8k). Taken together these data support the notion that cooperation between IRF5 and IRF8 promotes the aberrant accumulation, function, and differentiation of $\mathrm{ABCs}$ in $\mathrm{DKO}$ females. 
a

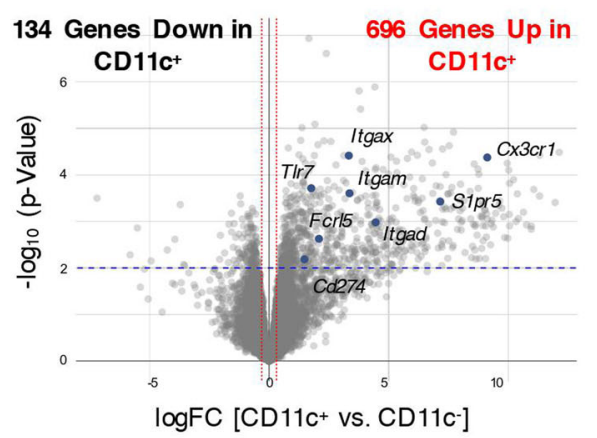

b

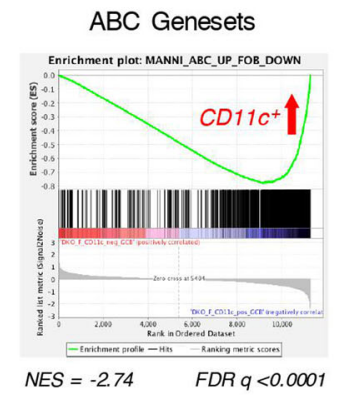

C

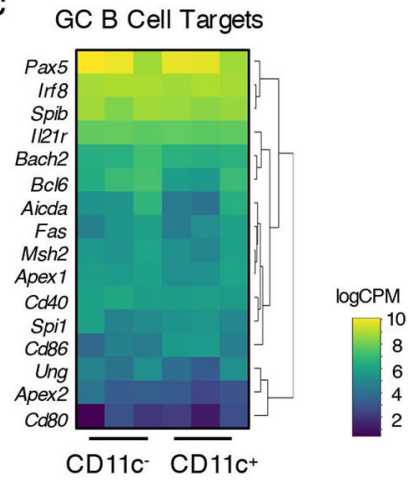

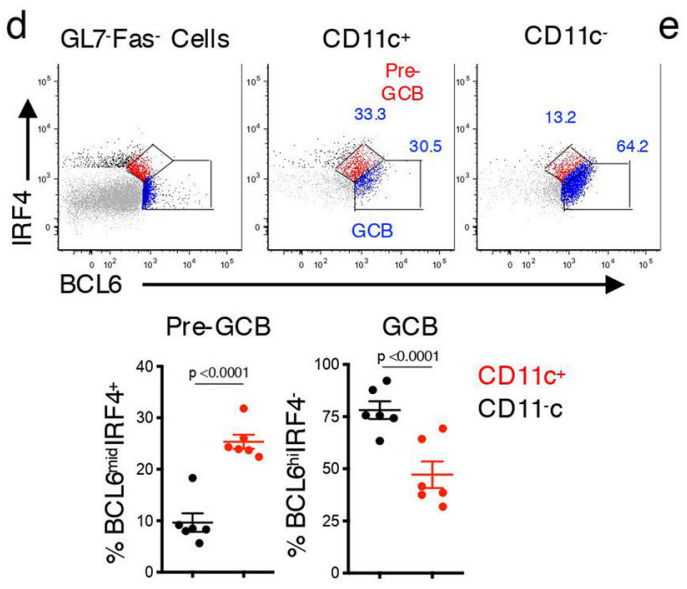

g
Top Enriched Pathways in CD11c+ vs. CD11C-

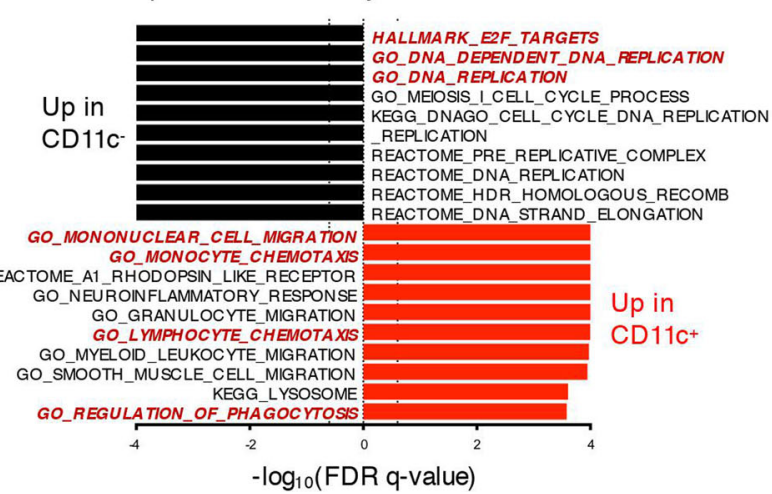
GO_DNA_DEPENDENT_DNA_REPLICATION GO_DNA_REPLICATION $\mathrm{h}$

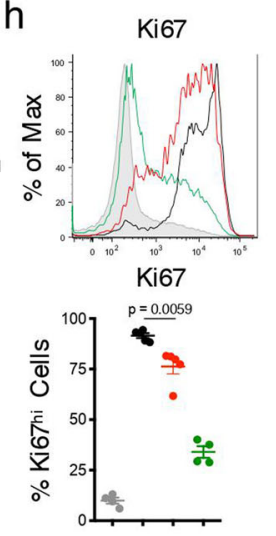

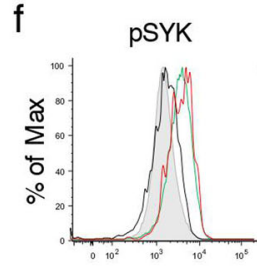

PSYK

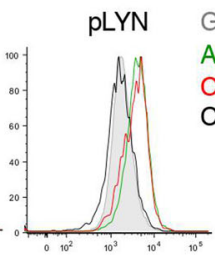

PLYN
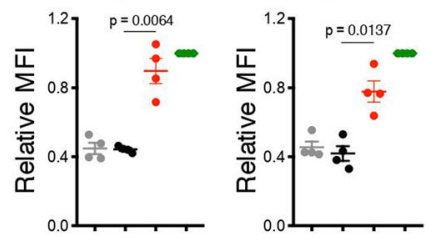
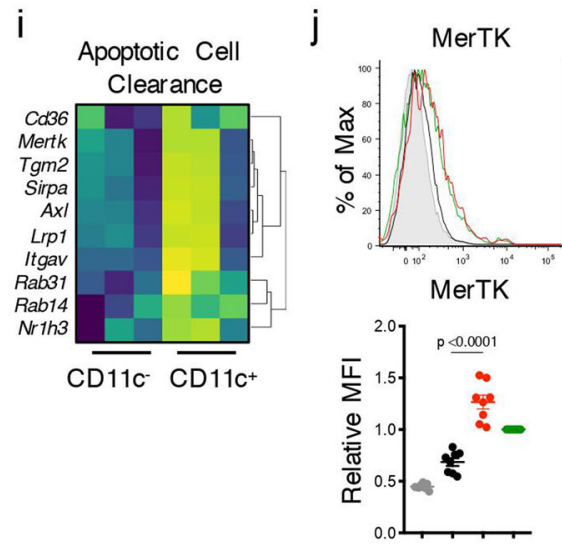

Fig. 5 CD11c ${ }^{+}$GC B cells exhibit a pre-GC B cell phenotype. RNA-seq was performed on sorted $C D 11 c^{+}$and $\mathrm{CD}_{11 \mathrm{c}^{-}} \mathrm{GL}^{+} \mathrm{CD}^{+} 8^{\mathrm{lo}} \mathrm{B}$ cells $\left(\mathrm{gated}\right.$ on $\mathrm{B} 22 \mathrm{O}^{+}$ splenocytes) from aged (24+wk) female DKO (F) mice. a Volcano plot showing genes differentially expressed $(p<0.01$ after Benhamini-Hochberg false discovery rate (FDR) was used to correct for multiple comparisons) between $\mathrm{CD} 11 \mathrm{c}^{+} \mathrm{B} 22 \mathrm{O}^{+} \mathrm{GL7} 7^{+} \mathrm{CD} 38^{\mathrm{lo}}\left(\mathrm{CD} 11 \mathrm{c}^{+}\right)$and $\mathrm{CD} 11 \mathrm{c}^{-} \mathrm{B} 22 \mathrm{O}^{+} \mathrm{GL7}+\mathrm{CD} 38^{\circ}$ (CD11c ${ }^{-}$) cells. b Plot showing the enrichment of the $A B C$ geneset from DKO mice in CD11c ${ }^{+} B 220^{+} G L 7^{+} C D 38$ lo cells. $\mathbf{c}$ Heatmap showing the expression of $G C$ B cell target genes in $\mathrm{CD} 11 \mathrm{c}^{+}$and $\mathrm{CD} 11 \mathrm{c}^{-} \mathrm{B} 22 \mathrm{O}^{+} \mathrm{GL} 7^{+} \mathrm{CD} 38^{\circ}$ cells. $\mathbf{d}$ Representative plots and quantifications of pre-GC B cells (BCL6 ${ }^{\text {mid }}$ IRF4 ${ }^{+}$) and $\mathrm{GC}$ B cells

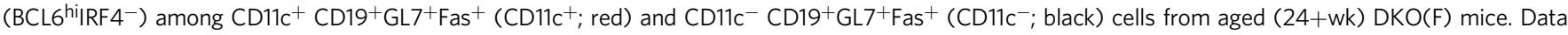
show mean $\pm S E M ; n=6$ from 2 independent experiments; $p$-value by paired two-tailed $t$-tests. e Plot showing the enrichment of a pre-GC geneset

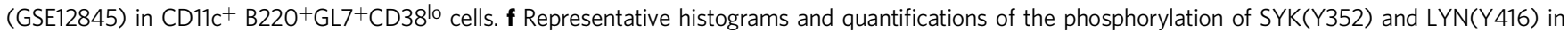

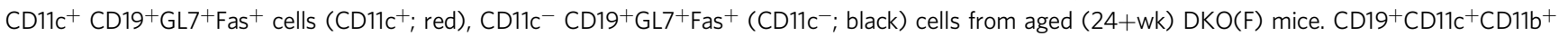
$\left(A B C s\right.$; green) $C_{19}{ }^{+} \mathrm{GL}^{-}-\mathrm{Fas}^{-}$cells (gray) are shown as control. Data show mean $\pm \mathrm{SEM} ; n=4$ from 3 independent experiments; $p$-value by paired twotailed $t$-test. $\mathbf{g}$ Plot showing the top pathways upregulated in CD11c $\mathrm{GL7}^{+} \mathrm{CD} 38^{\mathrm{lo}}$ cells (black) and $\mathrm{CD} 11 \mathrm{c}^{+} \mathrm{GL7}{ }^{+} \mathrm{CD} 38^{\circ}$ cells (red) by GSEA. Dotted line indicates significance threshold at FDR $q<0.25$. $\mathbf{h}$ Representative histogram and quantification of Ki67 expression in the indicated populations from DKO(F) mice. Data show mean $\pm S E M ; n=5$ from 3 independent experiments; $p$-value by paired two-tailed $t$-tests. $\mathbf{i}$ Heatmap showing the expression of genes related

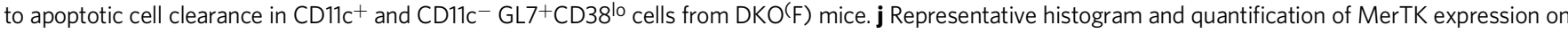
the indicated populations from aged (24+wk) DKO(F) mice. Data show mean \pm SEM; $n=8$ from 4 independent experiments; $p$-value by paired two-tailed $t$-tests. 
a

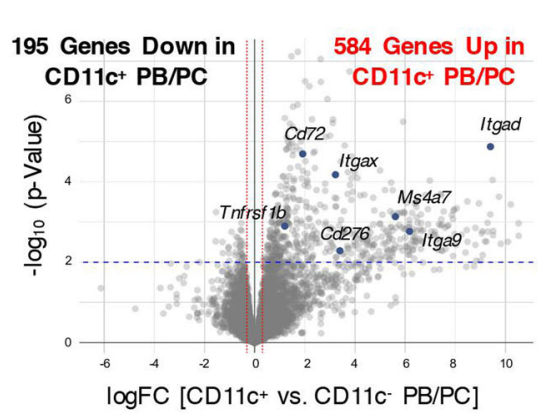

d

g

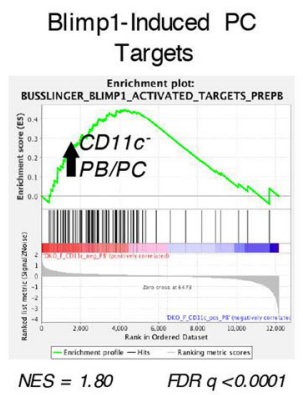

Top Enriched Pathways in CD11c+ PB/PC

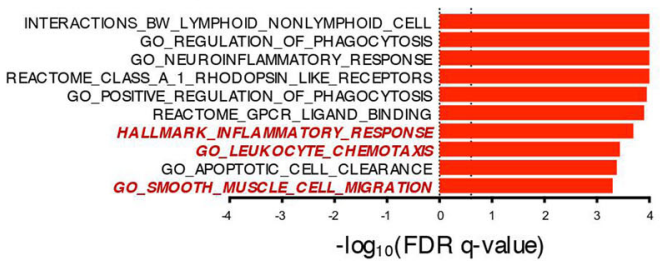

b

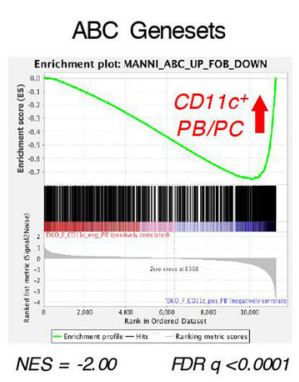

C
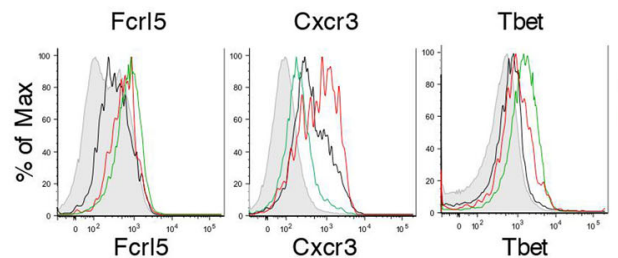

GL7-Fas- Cells

$\mathrm{ABC}$

CD11C+ $\mathrm{PB} / \mathrm{PC}$

CD11- PB/PC

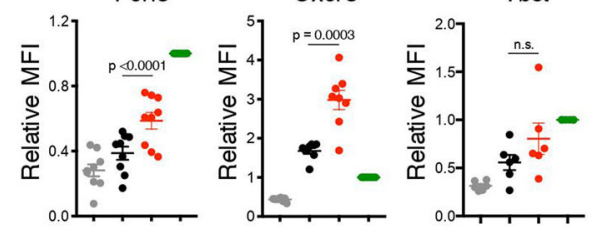

f

e

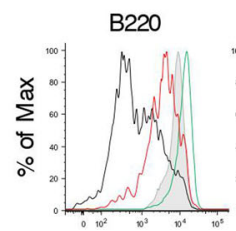

B220

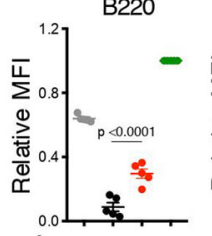

$\mathrm{h}$

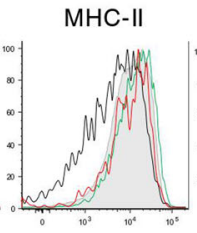

MHC-II
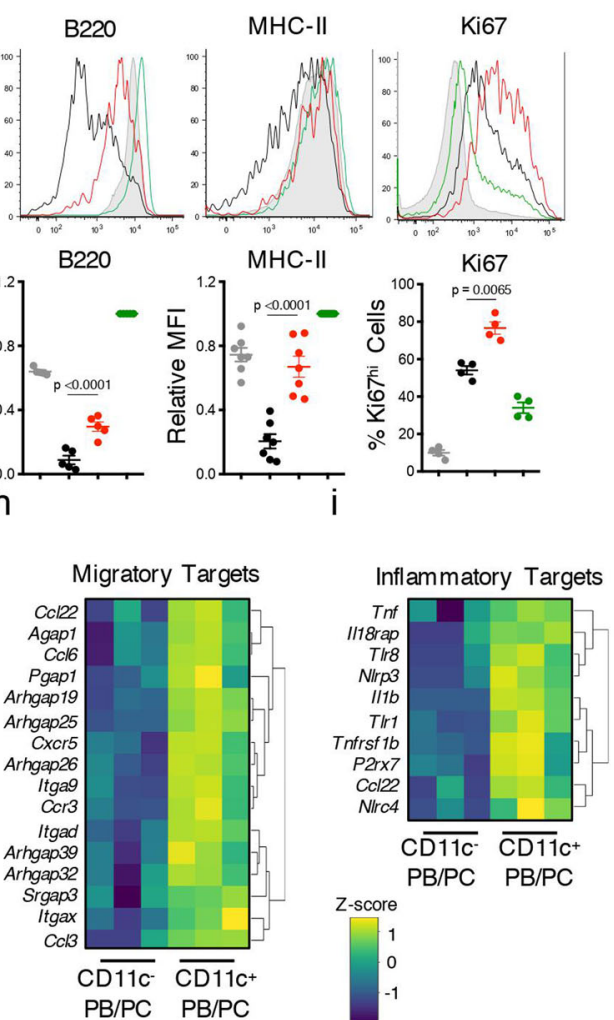

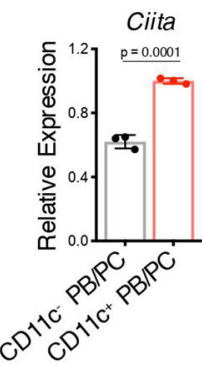

j

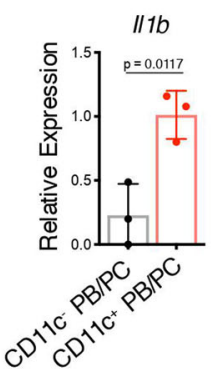

Fig. 6 Expansion of CD11c + PBs in DKO females. a RNA-seq was performed on sorted CD11c ${ }^{+}$and CD11c $-\mathrm{PB} / \mathrm{PCs}\left(\mathrm{CD} 138^{+} \mathrm{TACl}+\right)^{+}$from aged $(24+$ wk $)$ female DKO (F) mice. Volcano plot showing differentially expressed genes $(p<0.01$ after Benhamini-Hochberg false discovery rate (FDR) was used to correct for multiple comparisons) between $\mathrm{CD}_{11 c^{+}}$and $\mathrm{CD} 11 \mathrm{c}^{-} \mathrm{PB} / \mathrm{PCs}$. Blue dots indicate $\mathrm{ABC}$ target genes upregulated in CD11c ${ }^{+} \mathrm{PB} / \mathrm{PC}$. $\mathbf{b}$ Plot showing the enrichment of the $A B C$ geneset from DKO mice in CD11c + PB/PCs. c Representative histograms and quantifications of Fcrl5, Cxcr3, and Tbet

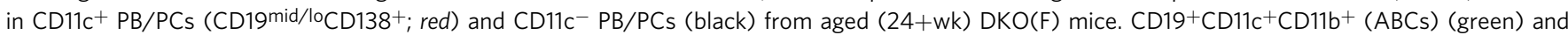
$\mathrm{CD}_{19}{ }^{+} \mathrm{GL} 7-\mathrm{Fas}^{-}$cells (gray) are shown as controls. Data show mean $\pm \mathrm{SEM} ; n=6$ for Tbet, $n=8$ for CXCR3, $n=9$ for FCRL5 over $3-5$ independent experiments; $p$-value by paired two-tailed $t$-tests. $\mathbf{d}$ Plots showing the enrichment of Blimp1-induced ${ }^{74}$ and IRF4-induced target genes in CD11 ${ }^{-}$PB/PCs by GSEA. e Representative histograms and quantifications of B220, MHC-II, and Ki67 expression in the indicated populations from aged (24+wk) DKO(F) mice. Data show mean \pm SEM; $n=4$ for Ki67, $n=5$ for B220, $n=7$ for MHC-II over 2-3 independent experiments; $p$-value by paired two-tailed $t$-tests. f Representative RT-qPCR showing Ciita expression in CD11c ${ }^{+}$or CD11c ${ }^{-}$PB/PCs sorted from aged (24+wk) DKO(F) mice as in Fig. 6a. Data representative of 3 independent experiments and show mean $\pm S D ; n=3 ; p$-value by unpaired two-tailed $t$-test. $\mathbf{g}$ Plot showing the top pathways enriched in $\mathrm{CD}_{11 c^{+}} \mathrm{PB} / \mathrm{PC}$ s as compared to CD11c ${ }^{-} \mathrm{PB} / \mathrm{PC}$ by GSEA. Dotted line indicates significance threshold at FDR $q<0.25$. $\mathbf{h}$, $\mathbf{i}$ Heatmap showing the expression of migratory $(\mathbf{h})$ and inflammatory target genes (i) that are differentially expressed $(p<0.01)$ between $C D 11 c^{+}$and CD11c $c^{-}$PB/PCs in DKO(F) mice from Fig. 6a. j Representative RT-qPCR showing I/1b expression in CD11c ${ }^{+}$or CD11c- PB/PCs sorted from aged (24+wk) DKO(F) mice as in Fig. 6a. Data representative of 3 independent experiments and show mean $\pm \mathrm{SD} ; n=3 ; p$-value by unpaired two-tailed $t$-test.

TLR7 duplication in DKO males promotes ABC dissemination and severe immunopathogenesis. While SLE preferentially affects females, males with lupus often exhibit a more rapid and severe course. Similarly, the shorter survival of Yaa-DKO males than DKO females suggested a more severe immunopathogenesis.
Interestingly, the early mortality and renal damage of Yaa-DKO males was accompanied by greater frequencies of $\mathrm{ABCs}$ in the blood and kidneys (Fig. 9a-c; Supplementary Fig. 8A, B). Furthermore, a histopathological analysis revealed that Yaa-DKO males also exhibited prominent inflammatory infiltrates in the 
a

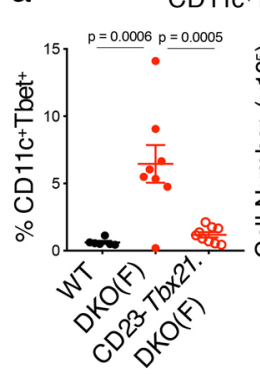

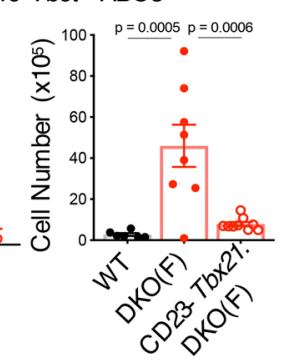

C

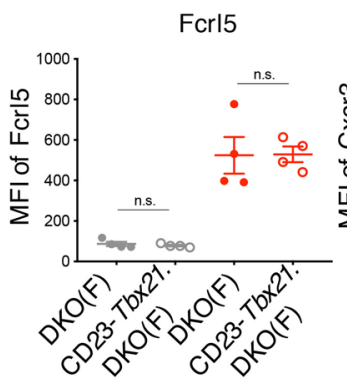

b

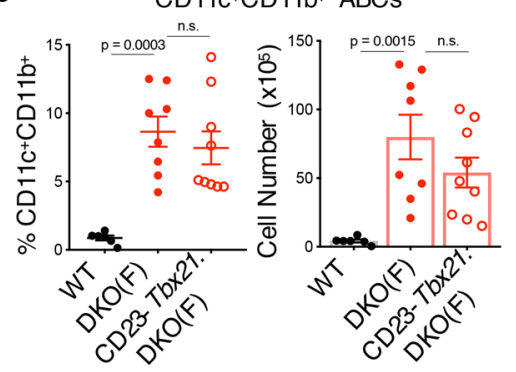

Cxcr3

\section{non-GCBs}

CD11c+CD11b+ $\mathrm{ABCs}$

d

Total GL7+Fas ${ }^{+}$Cells e

Total PB/PC

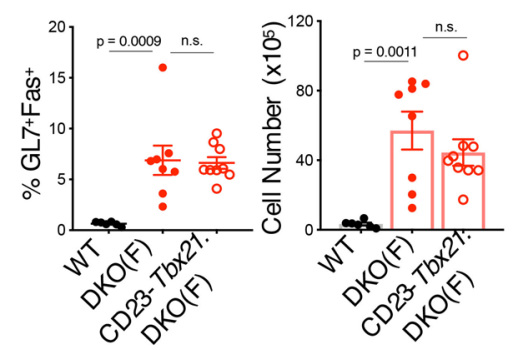

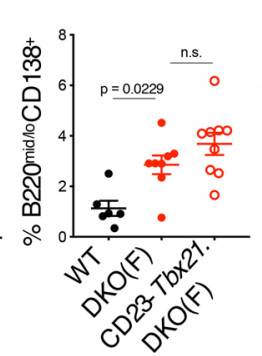

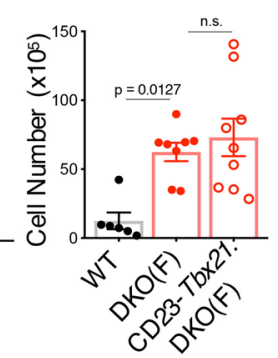

$f$

$\mathrm{CD}_{11 \mathrm{C}+} \mathrm{GL7}^{+} \mathrm{Fas}^{+}$Cells $\quad \mathrm{g}$

CD11C+ PB/PC
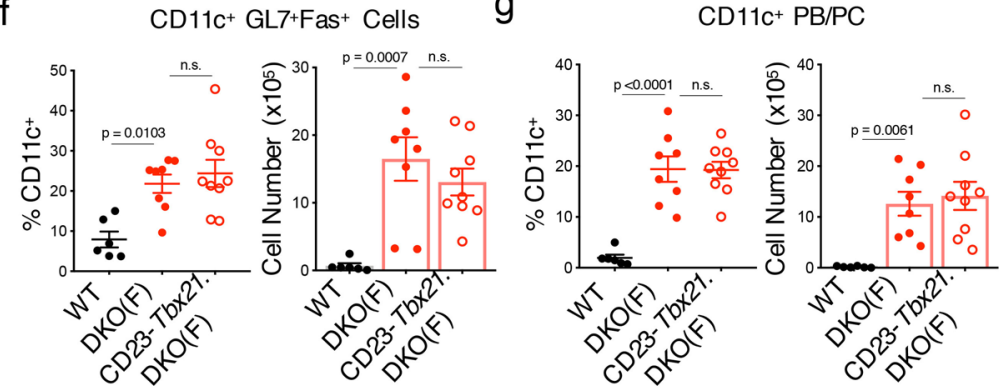

h

i
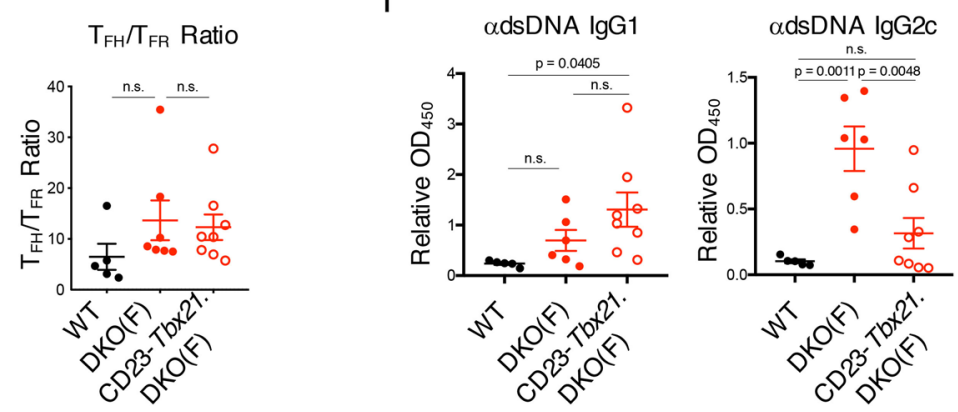

lungs although other organs, like the colon and the pancreas, were unaffected (Fig. 9d; Supplementary Fig. 8C). FACS analysis demonstrated that the lungs of Yaa-DKO males displayed a marked accumulation of $\mathrm{ABCs}, \mathrm{CD} 11 \mathrm{c}^{+}$and $\mathrm{CD} 11 \mathrm{c}^{-} \mathrm{GC} \mathrm{B}$ cells, and $\mathrm{CD}_{11 \mathrm{c}^{+}}$and $\mathrm{CD} 11 \mathrm{c}^{-} \mathrm{PB} / \mathrm{PC}$ as well as activated $\mathrm{T}$ cells (Fig. 9e; Supplementary Fig. 8D). DKO females also exhibited

lung inflammation, but as compared to age-matched Yaa-DKO males, the findings were less severe (Fig. 9d). Lung infiltrates in DKO females, however, worsened with age and were markedly ameliorated in aged CD23-Cre.Irf $8^{\text {flox/flox }}$ DKO females suggesting a crucial contribution of $\mathrm{ABCs}$ to the pulmonary inflammation (Fig. 9f). Thus, Tlr7-driven expansion of ABCs and their progeny 


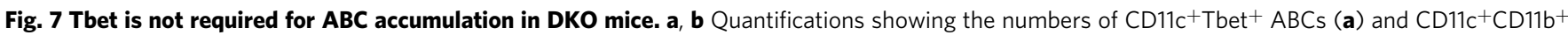
ABCs (b) from aged (24+wk) female C57BL/6 (WT; black circles), female DKO(F) (red circles), and female CD23-Cre.Tbx21/f.DKO (CD23-Tbx21.DKO (F)) mice (red open circles). Data show mean \pm SEM; $n=6$ for WT, $n=8$ for DKO(F), $n=9$ for CD23-Tbx21.DKO(F) over 6 independent experiments; $p$ value by 1-way ANOVA followed by Tukey's test for multiple comparisons. c Plots showing the MFI of Fcrl5 and Cxcr3 on the surface of CD11c $\mathrm{CD}^{+} 11 \mathrm{~b}^{+}$ $\mathrm{ABCs}$ (red) from the indicated mice. Non-GCBs (CD19+GL7-Fas ${ }^{-}$; gray) are shown as control. Data show mean $\pm \mathrm{SEM} ; n=4 ; p$-value by unpaired two-

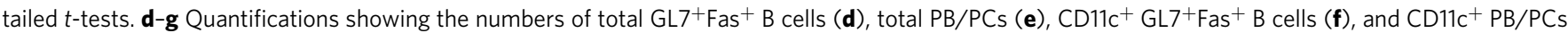
(g) from the indicated aged (24+wk) mice. Data show mean \pm SEM; $n=6$ for WT, $n=8$ for DKO(F), $n=9$ for CD23-Tbet.DKO(F) over 6 independent experiments; $p$-value by 1-way ANOVA followed by Tukey's test for multiple comparisons. $\mathbf{h}$ Quantifications showing the ratio of $T_{F H}$ to $T_{F R}$ cells ratio from the indicated mice. Data show mean \pm SEM; $n=5$ for WT, $n=7$ for DKO(F), $n=8$ for CD23-Tbet.DKO(F); $p$-value by 1-way ANOVA followed by Tukey's test for multiple comparisons. i Pooled ELISA data of anti-dsDNA IgG1 and IgG2c levels in the serum from the indicated aged (24+wk) mice. Data show mean \pm SEM; $n=5$ for WT, $n=6$ for DKO(F), $n=8$ for CD23-Tbet.DKO(F); $p$-value by 1-way ANOVA followed by Tukey's test for multiple comparisons.

can promote their accumulation in the lungs and the development of severe pulmonary inflammation.

Given that the marked lung inflammation in the setting of TLR7 dysregulation was reminiscent of the pathophysiology not only of SLE but also of severe viral infections such as COVID-19, we conducted additional hematologic and serologic analyses to assess whether other parameters known to be altered in this infection were similarly affected. A peripheral blood count demonstrated lower lymphocyte and platelet counts but increased monocytes in Yaa-DKO males than DKO males (Fig. 9g; Supplementary Fig. 8E). Yaa-DKO males also exhibited elevated levels of serum TNF and IL4 (Fig. 9h). We also assessed the production of antiphospholipid antibodies. While all DKOs produced higher levels of anti-phosphatidylserine (pS) IgM antibodies than WT controls, only Yaa-DKO males produced anti-pS IgG, anti-cardiolipin, and anti-MDA-LDL IgG (Fig. 9i). In line with the known ability of $\mathrm{ABCs}$ to produce antiphospholipid antibodies in response to pathogens and mediate hematologic abnormalities ${ }^{40}$, anti-pS IgG and anti-cardiolipin antibodies correlated with $\mathrm{ABC}$ and $\mathrm{PB} / \mathrm{PC}$ frequencies and there was a significant inverse association between $\mathrm{ABC}$ frequencies and platelet counts (Supplementary Fig. 8F-G). Thus, Tlr7 duplication in DKO males results in hematologic and serologic abnormalities that can be associated not only with SLE but also with severe viral infections like COVID-19.

To gain insights into the molecular features that might result in a more rapid and severe TLR7-induced immunopathogenesis in Yaa-DKO males than in DKO females, we compared the transcriptomes of $\mathrm{ABCs}$ and their progeny sorted from YaaDKO males with those of the corresponding populations sorted from DKO females. Only minimal differences were observed by GSEA between the ABCs of DKO females and those of Yaa-DKO males (Supplementary Fig. $8 \mathrm{H}$ ). However, a comparison of CD11c ${ }^{+}$pre-GC B cells and CD11 $c^{+}$PBs demonstrated that, as compared to DKO females, the populations derived from YaaDKO males were enriched for pathways involved in the production of antimicrobial peptides, cytokine interactions and signaling, and transcriptional regulation of granulopoiesis (Fig. 9j, k; Supplementary Fig. 8I-J). Thus, CD11 ${ }^{+}$effectors in Yaa-DKO males upregulate pathways that can enhance their pathogenicity and potentially enable their trans-differentiation.

\section{Discussion}

The mechanisms that underlie the sexual dimorphism observed in responses to infections and vaccinations, and in autoimmune diseases remain incompletely understood. Here, we delineate sexspecific differences in the function and differentiation of $\mathrm{ABCs}$, a subset of B cells that are emerging as critical mediators of antiviral responses and pathogenic players in autoimmunity. Using a spontaneous model of lupus where disease preferentially develops in females, we demonstrate that female ABCs exhibit a greater ability than male ABCs to accumulate, acquire an ISG signature, and further differentiate into effector populations, which include CD11 ${ }^{+}$pre-GC B cells and CD11c ${ }^{+}$PBs. BCR sequencing and fate mapping reveal oligoclonal expansion and relatedness amongst ABCs, GC B cells, and $\mathrm{PB} / \mathrm{PC}$ populations irrespective of the expression of CD11c. Genetic studies demonstrate a critical role for TLR7, IRF5, and IRF8 in promoting these abnormalities in females. Duplication of Tlr7 in males overrides the sex-bias and triggers severe immunopathology marked by intense lung inflammation and early mortality. Thus, sex-specific differences permeate several aspects of ABC biology in autoimmune settings suggesting that this compartment may be uniquely endowed to function in a sex-specific manner.

Our studies demonstrate marked differences in the ability of female and male DKO ABCs to express an ISG signature, which has recently been shown to be upregulated in ABCs and PCs from SLE patients and is one of the best-known features not only of this disease but also of antiviral responses ${ }^{7,41-43}$. The ISG signature observed in SLE patients indeed overlaps with that detected upon viral infections and immunizations and an inability to upregulate ISGs has recently been shown to distinguish severe from mild-to-moderate COVID-19 patients $^{44-46}$. Our epigenetic and genetic analyses furthermore indicate that this system is critically reliant on the dysregulation of IRF5 and IRF8 activity, well-known controllers of ISGs whose variants have long been associated with lupus pathogenesis and whose activity can be a target of viral evasion strategies ${ }^{46-48}$. While we cannot rule out that TLR7-driven IFNa production by pDCs could contribute to the differential expression of the ISG signature in DKO females and males, loss of repressive epigenetic markers at ISGs were selectively observed in ABCs, but not FoBs, from DKO females supporting the notion that acquisition of this signature is controlled in large part by cell-intrinsic mechanisms rather than exposure to an IFN-rich environment.

Sex-based differences were also observed in the ability of ABCs to further differentiate into effector subsets. Despite similar $\mathrm{T}_{\mathrm{FH}}$ responses to DKO males, DKO females exhibited a more robust expansion of GC B cells and PB/PCs, which in addition to classical $\mathrm{CD} 11 \mathrm{c}^{-}$subsets, also included $\mathrm{CD} 11 \mathrm{c}^{+}$ expressing subsets. BCR sequencing and fate mapping uncovered surprising relationships of $\mathrm{ABCs}$ not only with $\mathrm{CD} 11 \mathrm{c}^{+}$ pre-GC B cells and CD11 ${ }^{+}$PBs, but also with CD11c ${ }^{-}$GC B and $\mathrm{CD} 11 \mathrm{c}^{-} \mathrm{PC}$, which exhibited remarkably different transcriptional profiles from the $\mathrm{CD} 11 \mathrm{c}^{+} \mathrm{B}$ cell populations. The IRFs may again be a crucial component of this dysregulation. Indeed, the known ability of IRFs to homo/heterodimerize and target ISREs as well as interact with other transactivators like the Ets protein PU. $1^{47}$ may be well-suited to help confer a high degree of heterogeneity to ABCs and their progeny and finetune their transcriptional profiles. The pairing of IRF5 with IRF8 in this compartment may provide ABCs and their CD11c ${ }^{+}$ progeny with a more "innate" quality than traditional CD11c ${ }^{-} \mathrm{B}$ cell subsets and facilitate their distinctive combination of innate 
a

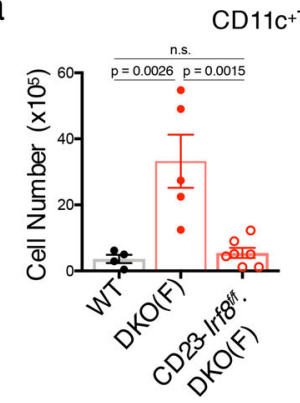

C

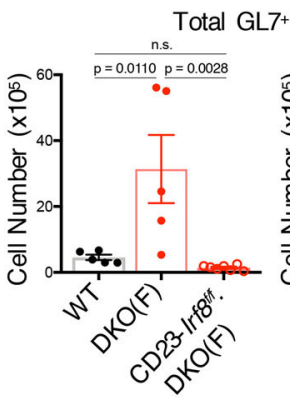

e

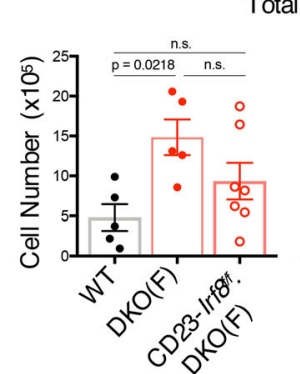

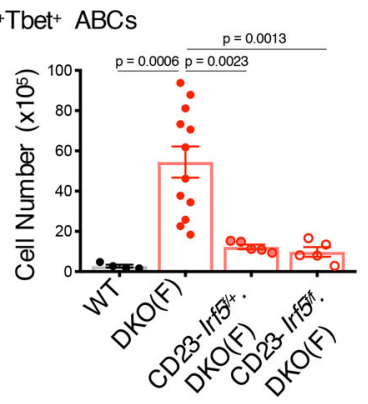

+ Fas $^{+}$Cells
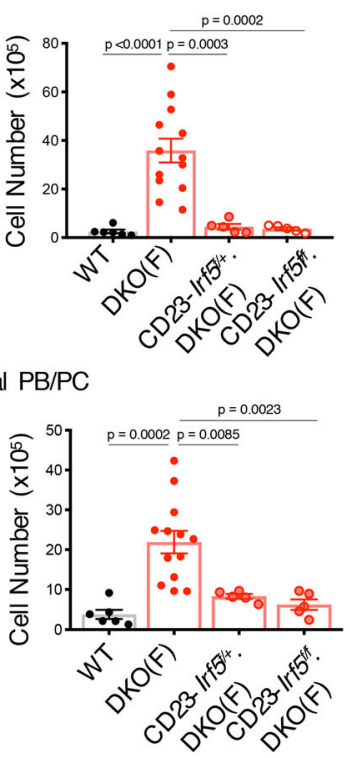

b

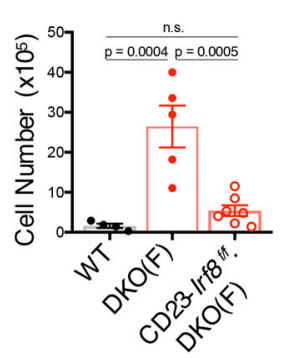

d

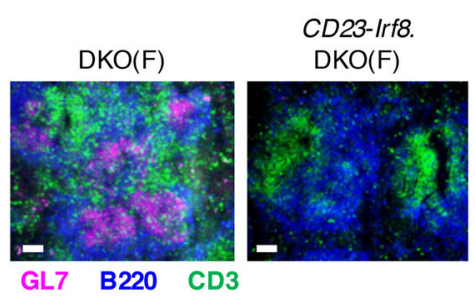

f

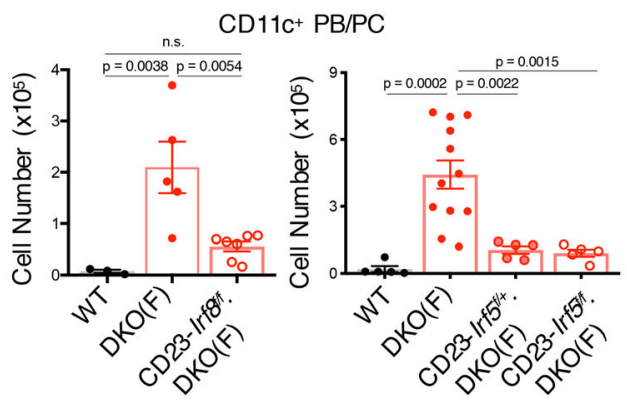

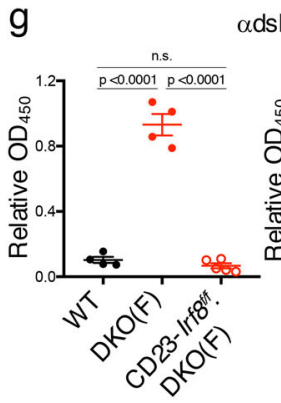

$\alpha d s D N A$ lgG2C

$\mathrm{h}$

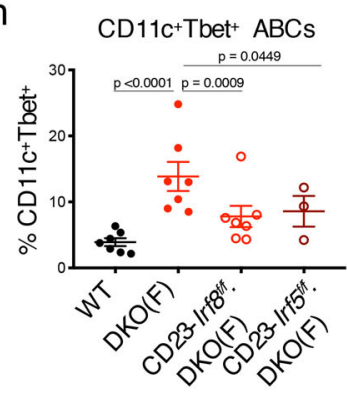

$\mathrm{k}$
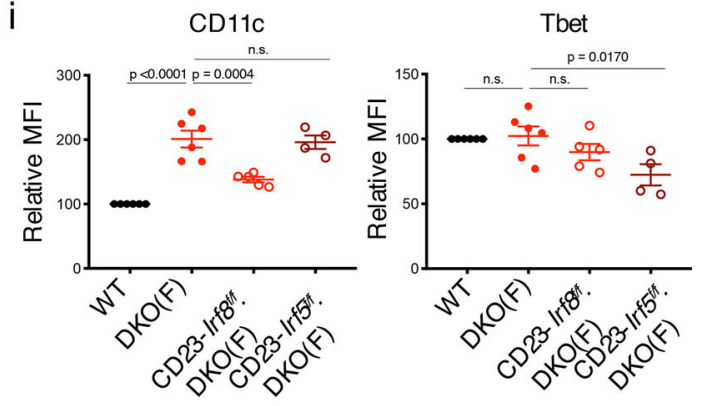

j

CXCL10 Production

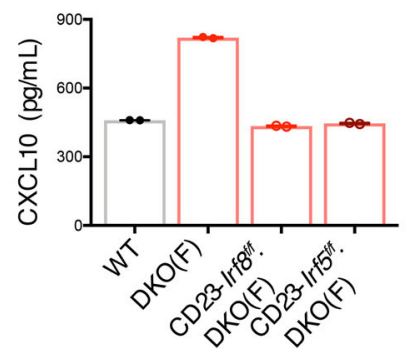

IRF8 ChIP

IRF5 ChIP

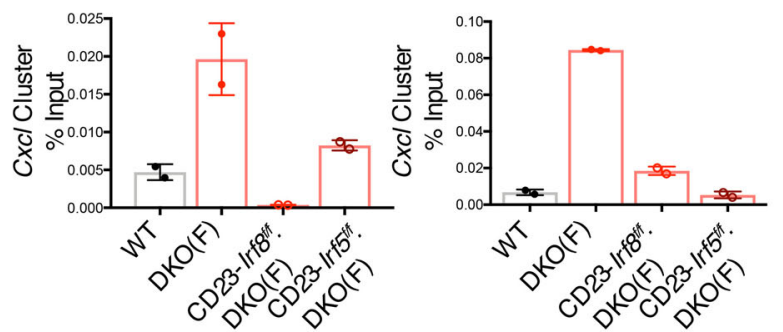

and adaptive functions. Changes in IRF8 activity, like those mediated by ROCK2 phosphorylation ${ }^{49}$, could instead result in the downregulation of typical $\mathrm{ABC}$ transcriptional targets like $\mathrm{CD} 11 \mathrm{c}$ and help promote its interaction with other transactivators enabling $\mathrm{ABCs}$ to give rise to both $\mathrm{CD} 11 \mathrm{c}^{+}$and $\mathrm{CD} 11 \mathrm{c}^{-}$ effector progeny. In support of this notion preliminary studies indicate that TLR7 engagement can inhibit ROCK2 activation thus directly affecting this balance.
In contrast to the marked effects observed upon deletion of IRF5 and IRF8, lack of B-cell T-bet in DKOs exerted more selective effects suggesting that, in this autoimmune setting, the generation and differentiation of $\mathrm{CD} 11 \mathrm{c}^{+} \mathrm{B}$ cells is less reliant on T-bet. T-bet however played a critical role in the acquisition of functional capabilities, such as the production of anti-dsDNA $\operatorname{IgG} 2 \mathrm{a} / \mathrm{c}$ antibodies, that have been associated with these cells. Given that a small fraction of FoBs was found to express T-bet in 
Fig. 8 IRF8 and IRF5 are required for ABC differentiation in DKO females. a-c Quantifications showing the numbers of CD11c ${ }^{+}$Tbet ${ }^{+} A B C s$ (a), $C D 11 C$ ${ }^{+}$CD11b + ABCs (b), and total GL7+Fas ${ }^{+}$B cells (c) from the spleens of aged (24+wk) female C57BL/6 (WT), female DKO(F), female CD23-Irf8.DKO(F), female CD23-Irf5f/+.DKO(F), and female CD23-Irfff/f.DKO(F) mice. Data show mean \pm SEM; $n=4$ for WT, $n=5$ for DKO(F), CD23-Irf5f/+.DKO(F), and CD23-Irf5.DKO (F), $n=7$ for CD23-Irf8.DKO(F), $n=12$ for DKO(F) over 6-7 independent experiments; $p$-value by 1-way ANOVA followed by Tukey's test for multiple comparisons. d Representative immunofluorescence images of B220 (blue), GL7 (pink), and CD3 (green) on the indicated mice. Data representative of 4 mice per genotype. Bars show $50 \mu \mathrm{m}$. e, $\mathbf{f}$ Quantifications showing the numbers of total PB/PCs (e; $\left.\mathrm{B}_{22} 2 \mathrm{mid}^{\mathrm{m} / \mathrm{lo}} \mathrm{CD} 138^{+}\right)$and $\mathrm{CD} 11 \mathrm{c}^{+} \mathrm{PB} / \mathrm{PCs}(\mathbf{f}) \mathrm{from}$ the indicated aged (24+wk) mice. Data show mean \pm SEM; $n=5 / 6$ for WT, $n=5 / 13$ for DKO(F), $n=7$ for CD23-Irf8.DKO(F), $n=5$ for CD23-Irf5 $5 /+$ (e); $n=3 / 5$ for WT, $n$ $=5 / 12$ for DKO(F), $n=7$ for CD23-Irf8.DKO(F), $n=5$ for CD23-Irf5/+ (f) over 6-7 independent experiments; $p$-value by 1-way ANOVA followed by Tukey's test for multiple comparisons. $g$ ELISA data showing anti-dsDNA IgG2c in the serum from the indicated aged (24+wk) mice. Data show mean \pm SEM; $n=4 / 8$ for WT, $n=4 / 14$ for DKO(F), $n=5$ CD23-Irf8.DKO(F), $n=7$ for CD23-Irf5+/-DKO(F), $n=6$ for CD23-Irf5/ff.DKO(F); $p$-value by 1-way ANOVA followed by Tukey's test for multiple comparisons. h-k CD23+ B cells from the indicated young (8-12wk) mice were stimulated for 2-3d with $\alpha \mid g M$, $\alpha C D 40$, and IL-21. h Quantification of CD11c ${ }^{+}$Tbet $^{+}$B cells after 3d culture. Data show mean \pm SEM; $n=7$ for WT, DKO(F), and CD23-Irf8.DKO(F), $n=3$ for CD23-Irf5.DKO(F); $p$ value by 1-way ANOVA followed by Tukey's test for multiple comparisons. i Quantifications showing the expression of CD11c and Tbet in the indicated strains after 3d culture as in Fig. 4h. Data show mean \pm SEM; $n=6$ for WT and DKO(F), $n=5$ for CD23-Irf8.DKO(F), $n=4$ for CD23-Irf5.DKO(F); $p$-value by 1-way ANOVA followed by Tukey's test for multiple comparisons. $\mathbf{j}$ ELISA data showing CXCL10 production in the supernatants after $3 \mathrm{~d}$ culture as in Fig. $4 \mathrm{~h}$. Data representative of 3 independent experiments and show mean \pm SD. k Representative ChIP-qPCR of IRF8 and IRF5 binding at the Cxcl cluster in the indicated young $(8-12 w k)$ mice. Data representative of 3 independent experiments and show mean \pm SD.

the fate mapping studies, additional analyses will furthermore be required to establish whether these $\mathrm{T}^{-}$bet $^{+}$cells are destined to acquire a full-fledged $\mathrm{ABC}$ phenotype or whether they represent a separate pool of T-bet ${ }^{+} \mathrm{B}$ cells that can also differentiate into a heterogenous progeny and are critical for autoantibody production.

VH sequencing demonstrated profound oligoclonal expansion and further confirmed common clonal relationships between ABCs, GC B cells, and PB/PCs, which again could be observed within both $\mathrm{CD} 11 \mathrm{c}^{+}$and $\mathrm{CD} 11 \mathrm{c}^{-}$compartments. Interestingly, different degrees of clonal overlap could be observed between $\mathrm{ABCs}$ and different progenies within distinct mice suggesting that while both extrafollicular and GC-like differentiation pathways may be available to these cells, the precise routes employed by $\mathrm{ABCs}$ to undergo terminal differentiation can vary depending on the specific inflammatory milieus that they are exposed to. Although the spontaneous GCs observed in this autoimmune setting displayed some atypical features, as evidenced by the finding that these GCs were exquisitely sensitive to the absence of IRF8 unlike those observed upon T-dependent immunization ${ }^{50}$, the ABC-derived GC populations did exhibit higher levels of SHM than ABCs suggesting that these GCs were functional. Notably, several of the $\mathrm{V}_{\mathrm{H}}$ regions overexpressed in DKO ABCs and their progeny, including $\mathrm{V}_{\mathrm{H}} 1$ (J558) and $\mathrm{V}_{\mathrm{H}} 14$ (SM7), have previously been associated with the production of lupus autoAbs and with the expanded ABCs of SLC ${ }^{-1-}$ mice, another spontaneous autoimmune model ${ }^{51}$.

The plasticity of ABCs and a limited capability to acquire GClike as well as $\mathrm{PB}$ phenotypes have been previously observed in Ehrlichia and influenza infection models $9,10,52$ suggesting that, upon encountering a pathogen, these differentiation pathways are available to ABCs, albeit in a restricted manner. One of the consequences of TLR7 overexpression in our autoimmune model furthermore was the dissemination of ABCs in the blood and their accumulation in the lungs, a pattern that was also transiently exhibited by $\mathrm{T}^{- \text {bet }^{+}} \mathrm{B}$ cells shortly after influenza infection ${ }^{9}$. Strikingly, the lung infiltrates in aged DKO females were markedly ameliorated in mice lacking IRF8 in B cells supporting a key role for $\mathrm{ABCs}$ and their progeny in promoting the pulmonary inflammation observed in DKOs. Thus, some of the pathogenesis of SLE may reflect a breakdown in the regulatory mechanisms aimed at restricting the differentiative routes and dissemination of $\mathrm{ABCs}$ in time and space. In this regard, the ability of the SWEF proteins to coordinate cytoskeletal organization and IRF function $^{21}$ may be particularly important. Indeed, by ensuring the proper positioning, cell-cell interactions, and transient migration of $\mathrm{ABCs}$ and by restricting the accessibility to IRF controlled transcriptional programs, these molecules could help ensure that a rapid initial response is coupled with the transient generation of a pool of progeny, which is diverse but limited in size.

Although lower levels of TLR7 responsiveness by male ABCs may protect DKO males from the development of lupus, the transcriptional profile of male ABCs showed enrichment for Rho GTPase signaling pathways. Dysregulation of these pathways has been linked to well-known disorders such as hypertension and cardiovascular disease, which also show a sexually dimorphic pattern but preferentially affect males ${ }^{53,54}$. This raises the intriguing possibility that lower levels of TLR7 engagement by male ABCs could not only result in suboptimal antiviral defenses but also promote a distinct set of pathogenic responses marked by vascular inflammation and thrombosis. Surprisingly, TLR7 duplication in Yaa-DKO males resulted in more profound immunopathogenesis than that observed in DKO females suggesting that females may have evolved mechanisms to contain the overwhelming inflammatory effects that may accompany the greater levels of TLR7 stimulation that they are predisposed to due to incomplete XCI. In this regard, it is interesting to note that both our studies and recent work in humans suggest that $\mathrm{ABCs}$ may be uniquely susceptible to partially escape $\mathrm{XCI}^{55}$. We cannot, however, rule out that the differences between Yaa-DKO males and DKO females primarily result from complete rather than partial escape from XCI secondary to the translocation of the Xchromosome segment onto the Y-chromosome. While our studies have highlighted a crucial role for TLR7 in mediating the sex differences in the development of lupus in this model, sex hormones like estrogen and androgen have also been shown to regulate $B$ cell responses including their positioning and survival and to modulate disease pathogenesis ${ }^{56,57}$ and thus are also important contributors to these differences.

Intriguingly, several of our findings are reminiscent of the pathophysiology of COVID-19 where disease outcomes have shown striking age- and sex-dependent differences ${ }^{1}$. Indeed, expansion of T-bet ${ }^{+} \mathrm{B}$ cells (including activated naïve and $\mathrm{DN}$ ) and $\mathrm{PBs}$, which in some cases can exhibit reprogramming potential and lower levels of SWAP-70, have all been observed in severe COVID-19 patients ${ }^{12,13,58,59}$. Some of the features known to accompany disease severity in COVID-19 such as lymphopenia, thrombocytopenia, production of antiphospholipid antibodies, and a broad array of cytokine responses ${ }^{60,61}$ could be observed in Yaa-DKO males in the absence of any viral infections suggesting that these effects could directly result from unbridled TLR7 stimulation. Whether the reported ability of ABC-like cells 
a

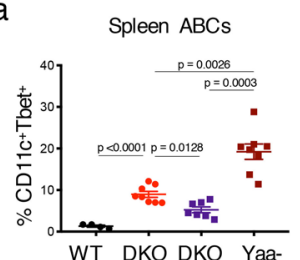

(F) (M) DKO(M)

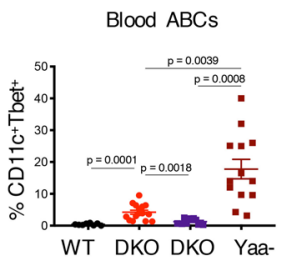

(F) (M) $\mathrm{DKO}(\mathrm{M})$

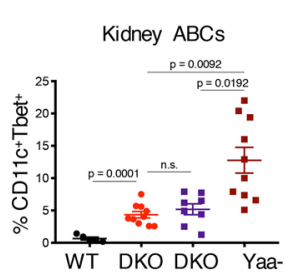

(F) (M) $\mathrm{DKO}(\mathrm{M})$ b

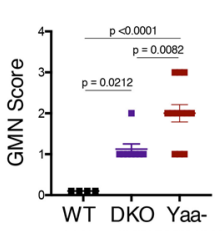

(M) (M)DKO(M)
C

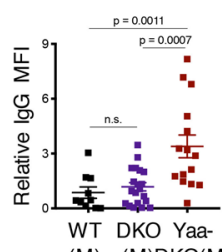

(M) (M)DKO(M)

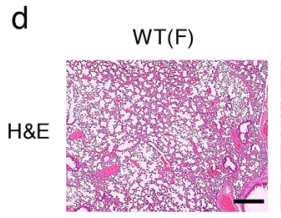

$\mathrm{DKO}(\mathrm{F})$

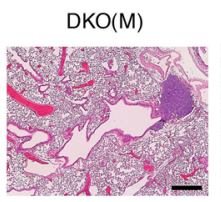

Yaa-DKO(M)
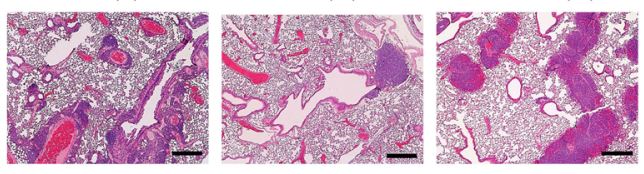

e

Lung $A B C s$

Total GL7+Fas + Cells

$\mathrm{CD}_{11 \mathrm{C}^{+} \mathrm{GL7}+\mathrm{Fas}}{ }^{+}$Cells

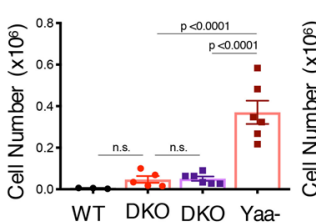

(F) (M) DKO

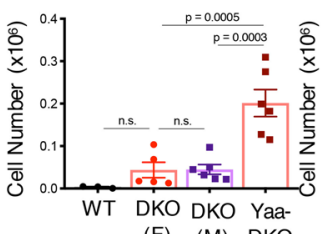

(F) (M) DKO
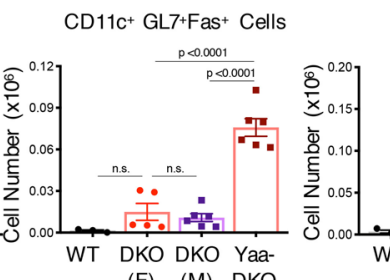

Total PB/PC
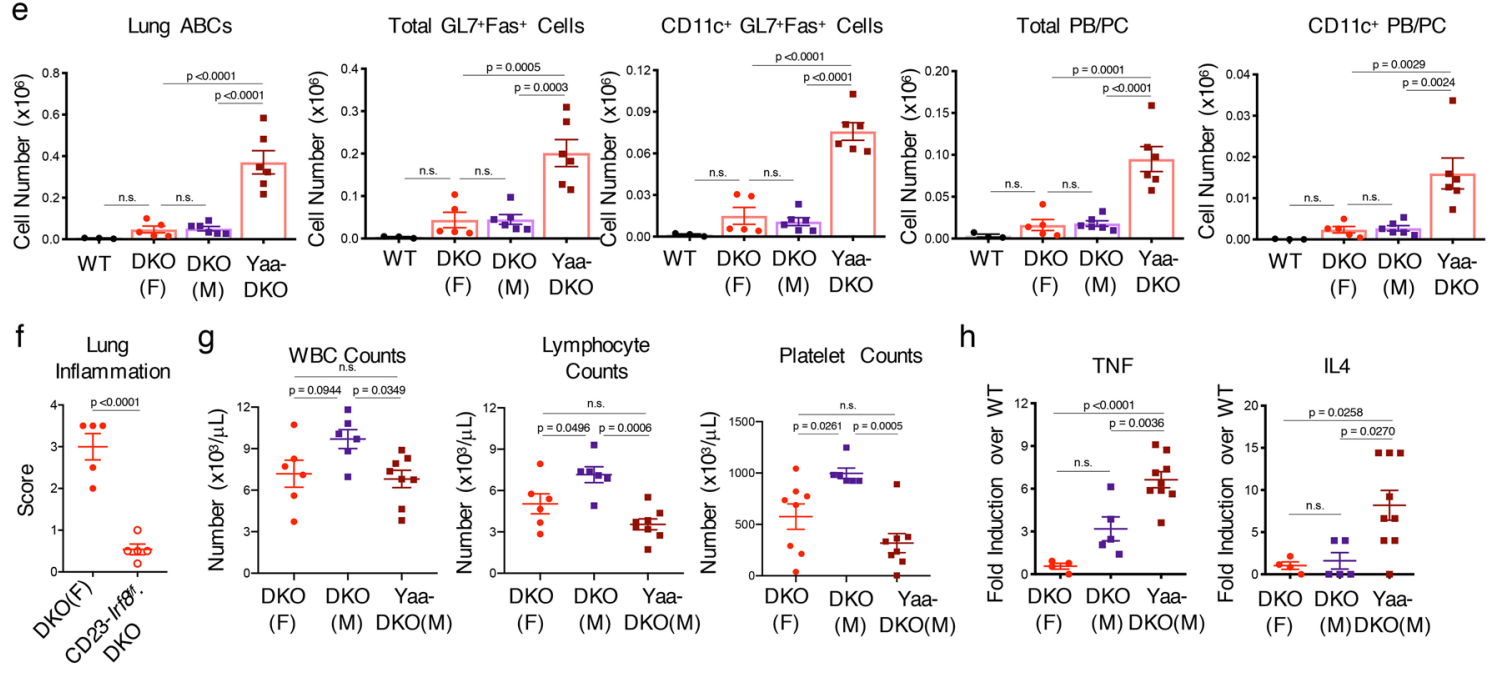

(F) (M) DKO

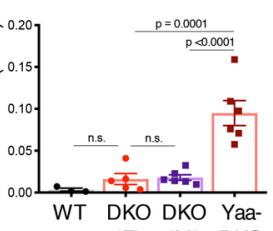

(F) (M) DKO

(F) (M) DKO

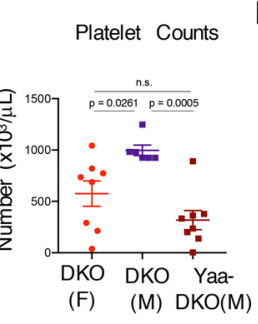

$\mathrm{h}$
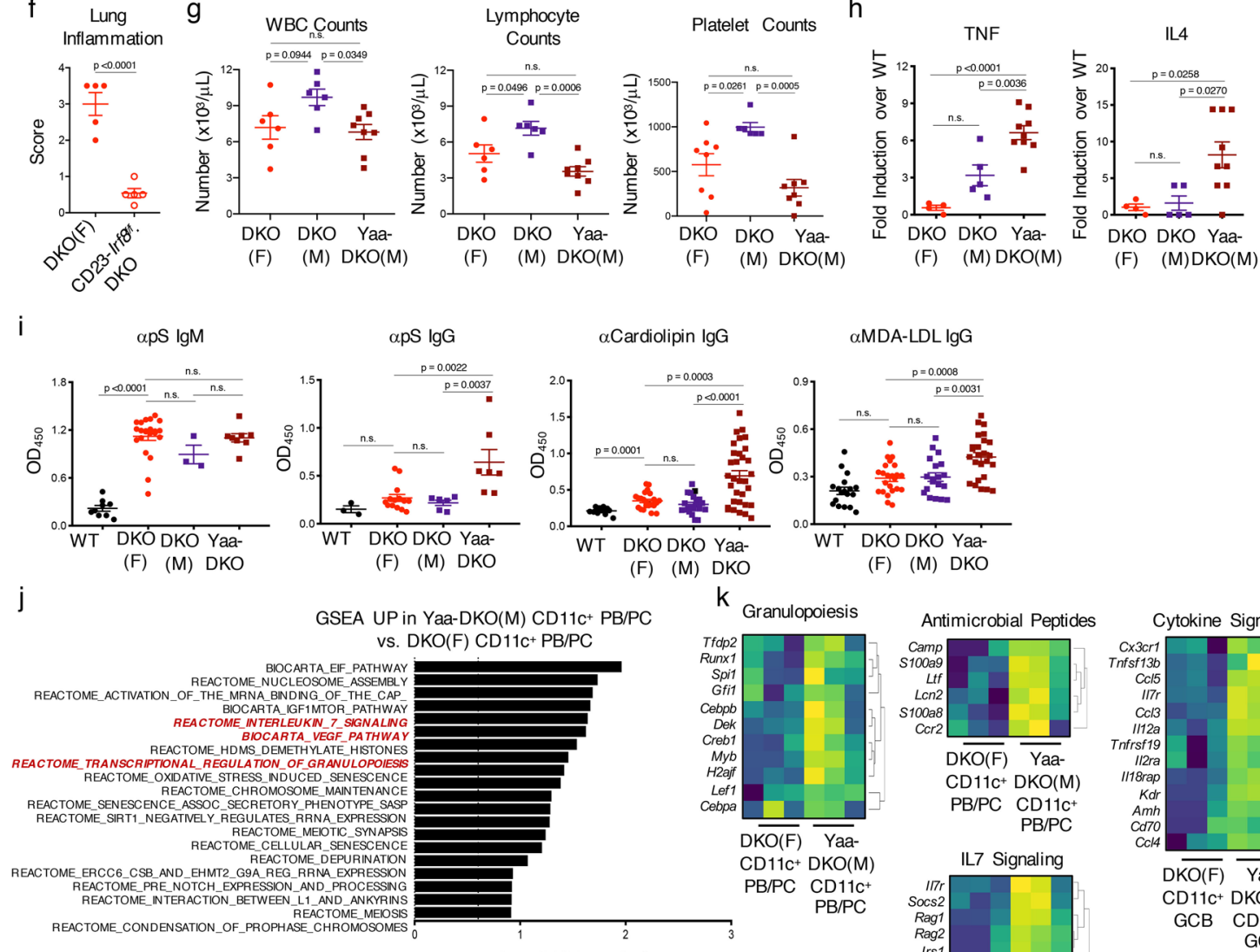

$-\log _{10}$ (FDR q-value)

to accumulate with age in adipose tissue in an IL-1-dependent manner ${ }^{62}$ could provide a ready depot of these cells and lower the threshold of TLR7 stimulation needed by these cells to acquire full pathogenic functions will be an important question to be addressed given the link between obesity and COVID-19 outcomes. The marked sex differences in the pathways utilized by female and male $A B C$ subsets suggest that the effectiveness of approaches targeting these cells may demonstrate sex-dependent differences. Such a scenario may be encountered, for instance, in the case of statins or geranylgeranylation inhibitors that can inhibit Rho-GTPases and have vaccine adjuvant properties ${ }^{63}$. Sex-specific differences in $\mathrm{ABC}$ function and differentiation could 
Fig. 9 TIr7 duplication promotes ABC dissemination and immunopathogenesis. a Quantification of CD11c ${ }^{+}$Tbet ${ }^{+}$ABCs from the spleens, blood, and kidneys of aged (24+wk) female C57BL/6 (WT; black circles), female DKO(F) (red circles), male DKO(M) (purple squares), and male Yaa-DKO(M) (maroon squares) mice. Data shows mean $\pm \mathrm{SEM} ; n=4 / 8 / 5$ for WT, $n=8 / 15 / 10$ for DKO(F), $n=7 / 12 / 8$ for DKO(M), $n=8 / 13 / 10$ for Yaa-DKO(M) over 5 independent experiments; $p$-value by 1-way Brown-Forsythe and Welch ANOVA followed by Games-Howell's test for multiple comparisons. b Glomerulonephritis (GMN) scores of kidneys from the indicated aged (24wk+) mice. Data shows mean \pm SEM; $n=4$ for WT, $n=8$ for DKO(M), $n=12$ for Yaa-DKO(M); $p$-value by 1-way ANOVA followed by Tukey's test for multiple comparisons. c Quantification of lgG deposition in the kidneys from the indicated aged mice. Data shows mean \pm SEM; $n=10$ fields across 2 mice for WT, $n=20$ fields across 4 mice for $\operatorname{DKO}(M), n=15$ fields across 3 mice for Yaa-DKO(M); $p$-value by 1-way ANOVA followed by Tukey's test for multiple comparisons. d Representative H\&E images of lungs from the indicated aged (30wk+) mice. Data representative of $2 \mathrm{WT}, 4 \mathrm{DKO}(\mathrm{F}), 4 \mathrm{DKO}(\mathrm{M})$, and

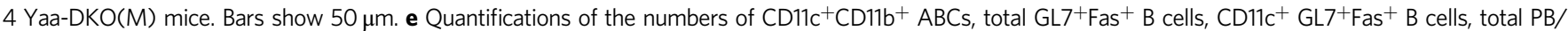
$\mathrm{PCs}$, and $\mathrm{CD} 11 \mathrm{c}^{+} \mathrm{PB} / \mathrm{PCs}$ in the lungs from the indicated aged (24wk+) mice. Data show mean $\pm \mathrm{SEM} ; n=3$ for WT, $n=5$ for DKO(F), $n=6$ for DKO(M) and Yaa-DKO(M) over 2 independent experiments; $p$-value by 1-way ANOVA followed by Tukey's test for multiple comparisons. f Plot showing the inflammation score in the lungs of $45 \mathrm{wk}$-old female CD23-Cre.Irf $8^{f / f} . \mathrm{DKO}(\mathrm{F})$ and female Irf $8^{f / f}$.DKO(F) control mice as determined by H\&E staining. Data show mean $\pm \mathrm{SEM} ; n=5$; $p$ value by unpaired two-tailed $t$-test. $\mathbf{g}$ Plots showing the numbers of white blood cells, lymphocytes, and platelets in the blood from the indicated aged (24wk+) mice. Data show mean \pm SEM; $n=6$ for DKO(F) and DKO(M), $n=8$ for Yaa-DKO(M); $p$-value by 1-way ANOVA followed by Tukey's test for multiple comparisons. $\mathbf{h}$ Luminex data showing the fold-increase in serum levels of TNF and IL4 in aged (24wk+) DKO(F), DKO(M), and Yaa-DKO(M) mice relative to WT. Data show mean \pm SEM; $n=4$ for DKO(F), $n=5$ for DKO(M), $n=9$ for Yaa-DKO(M); $p$-value by 1-way ANOVA followed by Tukey's test for multiple comparisons. i ELISA data for anti-phosphatidylserine (pS) IgM and $\operatorname{lgG}$, anti-Cardiolipin IgG, and anti-MDA-LDL IgG in the serum from the indicated aged $(24 \mathrm{wk}+)$ mice. Data show mean $\pm \mathrm{SEM}$; for anti-pS IgM, $n=9$ for WT, $n=22$ for DKO(F), $n=3$ for DKO(M), $n=8$ for Yaa-DKO(M); for anti-pS IgG $n=3$ for WT, $n=14$ for DKO(F), $n=6$ for DKO(M), $n=7$ for Yaa-DKO(M); for anti-Cardiolipin lgG, $n=11$ for WT, $n=23$ for DKO(F), $n=20$ for DKO(M), $n=32$ for Yaa$\operatorname{DKO}(\mathrm{M})$; for anti-MDA-LDL IgG, $n=18$ for WT, $n=23$ for DKO(F), $n=19$ for DKO(M), $n=25$ for Yaa-DKO(M); $p$-values by 1-way ANOVA followed by Tukey's test for multiple comparisons. j RNA-seq was performed on sorted CD11c ${ }^{+} \mathrm{PB} / \mathrm{PCs}\left(\mathrm{CD} 138^{+} \mathrm{TACl}^{+}\right)$from Yaa-DKO(M) mice as in Fig. 6a. Plot shows top pathways enriched in CD11 $c^{+} \mathrm{PB} / \mathrm{PC}$ f from Yaa-DKO(M) mice as compared to CD11c PB/PCs from DKO(F) mice as determined by GSEA. Dotted line indicates significance threshold at FDR q $<0.25$. $\mathbf{k}$ Heatmaps showing the expression of genes enriching the granulopoiesis, antimicrobial peptide, cytokine signaling, and IL7 signaling genesets in Yaa-DKO CD11c ${ }^{+}$PB/PCs.

thus not only contribute to the well-known sex-bias that underlies several autoimmune diseases but also broadly impact responses to pathogens and vaccination.

\section{Methods}

Mice. All mice used in this study were on a C57BL/6 background. DEF6-deficient $\left(D e f \sigma^{\text {tr/tr}}\right)$ mice were generated by Lexicon Pharmaceuticals, Inc. using a genetrapping strategy. Integration of the gene-trapping construct occurred in the first intron of the Def6 gene, downstream of the exon coding for the initiation methionine. Targeted ES cells (129SvEv) were injected into C57BL/6 blastocysts to generate chimeric mice, which were then backcrossed to C57BL/6 mice for $>10$ generations ${ }^{22}$. Swap70-deficient $\left(\right.$ Swap $\left.70^{-1-}\right)$ were generated via a targeting construct designed to replace a $2.7-\mathrm{kb} \mathrm{SacI} / \mathrm{SmaI}$ fragment containing exon 1 of the SWAP-70 gene with a phosphoglycerate kinase (PGK)-neo resistance cassette ${ }^{22}$. Def6 ${ }^{\mathrm{tr} / \mathrm{tr}}$ Swap $70^{-1-}$ (DKO) mice were generated by crossing Def6 $6^{\mathrm{tr} / \mathrm{tr}}$ mice with Swap $70^{-1-}$ mice that had been backcrossed onto C57BL/6 background for $>10$ generations $^{22}$. C57BL/6 mice, Irff ${ }^{\mathrm{f} / \mathrm{f}}$ mice (\#014175), and Tbx21/f/ mice (\#022741) were from Jackson Laboratory. B6.SB-Yaa/J were originally provided by Derry Roopenian and are available through Jackson (Yaa; \#000483), Tlr7-deficient were originally provided by Eric Pamer and are available through Jackson $\left(T l r 7^{-l-}\right.$ $\# 008380)^{64}$. Male Yaa mice were crossed with female DKO mice to generate YaaDKO male mice ${ }^{65}$. Irff f/f mice, Tbx2 $1^{f / f}$ mice, and $T l r 7^{-1-}$ mice were crossed with DKO mice to generate $I r f 8^{f / f}$.DKO, $T b \times 21^{f / f}$.DKO mice, and $T l r 7^{-l-}$.DKO mice. Generation of DKO Blimp1-YFP and Irf5/f DKO mice were previously described ${ }^{20,66}$. CD23-cre mice were provided by Jayanta Chaudhuri and were previously described ${ }^{67}$. Tbet-zsGreen-T2A-CreER ${ }^{T 2}$-Rosa26-loxP-STOP-loxPtdTomato DKO (ZTCE-DKO) mice were generated by crossing DKO mice with Tbet-zsGreen-T2A-CreER ${ }^{\mathrm{T} 2}$ provided from Jinfang $\mathrm{Zhu}^{36}$ and with B6.Cg-Gt

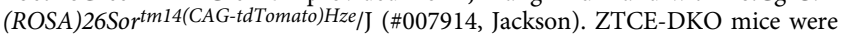
treated with Tamoxifen by oral gavage $3 \mathrm{~d}$ before experiments were conducted. All mice used in the experiments were kept under specific pathogen-free conditions. Control animals for most experiments were bred separately, but co-housed. Experiments comparing $\mathrm{DKO}(\mathrm{M})$ mice with Yaa-DKO(M) mice were performed with animals that were not co-housed. Mice were euthanized using carbon dioxide. All animal experiments were approved by the Institutional Animal Care and Use Committee of the Hospital for Special Surgery and WCMC/MSKCC and the experiments were carried out following these established guidelines.

Antibodies and flow cytometry. The following monoclonal antibodies to mouse proteins were used for multi-parameter flow cytometry: B220-PB or B220-APC/ Cy7 (RA3-6B2; 1:400), CD4-PerCP/Cy5.5 (RM4-5; 1:400), CD45-PerCP/Cy5.5 (HI30; 1:400), CD11b-PE/Cy7 or CD11b-FITC (M1/70; 1:400), CD11c-APC/Cy7 or CD11c-APC (N418; 1:400), CD19-PB or CD19-PE (HIB19; 1:400), CD21-APC (7E9; 1:200), CD23-PE or CD23-PerCP/Cy5.5 (B3B4; 1:200), CD44-PE/Cy7 or CD44-A700 (IM7; 1:200), CXCR3-A488 (CXCR3-173; 1:200), MHC-II-PerCP/ Cy5.5 (AF6-120.1; 1:600), and Tbet-PE or Tbet-PE/Cy7 (4B10; 1:800) were obtained from BioLegend. Streptavidin-conjugated antibodies were also obtained from BioLegend. Antibodies to BCL6-PE or BCL6-PE/Cy7 (K112-91; 1:100), CD138-APC (281-2; 1:1200), CXCR5-Biotin (2G8; 1:200), Fas-Biotin (Jo2; 1:200), FcRL5-FITC (509F6; 1:200), and GL7-FITC (1:600) were obtained from BD. Antibodies to Foxp3-APC (FJK-16s; 1:100), IgD-FITC (11-26; 1:500), IgM-PE/Cy7 (II/41; 1:1000), IRF4-FITC (3E4; 1:200), IRF8-PE (V3GYWCH; 1:200), Ki67-PE (solA15; 1:800), MerTK-APC (DS5MMER; 1:200), and PD1-PB (J43; 1:200) were obtained from eBioscience. For intracellular staining, cells were fixed after surface staining at $4{ }^{\circ} \mathrm{C}$ with the Transcription Factor Staining Kit (eBioscience; \#00-552300) following the manufacturer's instructions. For intracellular cytokine staining, splenocytes were stimulated with $50 \mu \mathrm{g} / \mathrm{mL}$ PMA and $1 \mu \mathrm{M}$ Ionomycin for $4 \mathrm{hr}$. Cells were incubated with BrefeldinA for the final $3 \mathrm{~h}$ of stimulation. After stimulation, cells were fixed and permeabilized with a Transcription Factor Staining Kit (eBioscience; \#00-5523-00) and stained using anti-IFN $\gamma$-APC (BioLegend; XMG1.2; 1:200) and recombinant mouse IL21R Fc Chimera (R\&D; 1:600) followed by PE-labeled affinity-purified $\mathrm{F}\left(\mathrm{ab}^{\prime}\right)_{2}$ fragment of goat anti-human $\mathrm{Fc} \gamma$ (Jackson ImmunoResearch). For detection of phosphorylated antigens, splenocytes were fixed in BD Fixation Buffer (\#554714) for $20 \mathrm{~min}$ at RT. Cells were then washed and permeabilized in $90 \%$ methanol for $30 \mathrm{~min}$ at $-20^{\circ} \mathrm{C}$ and then incubated with antibodies against phosphorylated SYK (Y352; Cell Signaling) or LYN (Y416; Cell Signaling) for $45 \mathrm{~min}$ at room temperature. LysoTracker staining was done before surface staining with $20 \mathrm{nM}$ LysoTracker-Deep Red (Thermo; L12492) for $45 \mathrm{~min}$ at $37^{\circ} \mathrm{C}$ in RPMI 1640 . All data were acquired on a BD FACS Canto and analyzed with FlowJo (TreeStar) software.

Cell sorting. Single-cell suspensions were prepared from spleens of the indicated mice. For sorting ABCs, splenocytes were pre-enriched for B cells with B220 microbeads (Miltenyi Biotec; \#130-049-501) following manufacturer's instructions. B cells were stained with CD11c-APC (N418; 1:400), CD11b-FITC (M1/70; 1:400), CD19-PB (HIB19; 1:400), B220-APC/Cy7 or B220-PE/Cy7s (RA3-6B2; 1:400), and CD23-PE (B3B4; 1:200) and were sorted on FACS Aria or Influx (BD). ABCs were collected for RNA or cultured for 7d with $1 \mu \mathrm{M}$ Imiquimod in RPMI 1640 medium (Corning) supplemented with 10X FBS (Atlanta Biologicals), $100 \mathrm{U} / \mathrm{mL}$ Penicillin (Corning), $100 \mathrm{mg} / \mathrm{mL}$ Streptomycin (Corning), $1 \mathrm{X}$ non-essential amino acids (Corning), 2mM s-Glutamine (Corning), $25 \mathrm{mM}$ HEPES (pH7.2-7.6; Corning), and $50 \mu \mathrm{M} \beta$-Mercaptoethanol. For sorting CD11 $\mathrm{c}^{+}$and CD11c ${ }^{-}$GC B-like cells and $\mathrm{PB} / \mathrm{PCs}$, splenocytes were pre-enriched using biotinylated antibodies against B220 and CD138 and with streptavidin-conjugated microbeads (Miltenyi Biotec; \#130-048-101) following manufacturer's instructions. Cells were stained with CD11c-APC/Cy7 (N418; 1:400), CD19-PB (HIB19; 1:400), CD138-APC (281-2; 1:600), CD38-PE/Cy7 (90; 1:600), GL7-FITC (1:600), and TACI-PE (8F10; 1:400) and were sorted on FACS Aria or Influx (BD).

B cell cultures. $\mathrm{CD} 23^{+} \mathrm{B}$ cells were purified from single cell suspensions of splenocytes with biotinylated anti-CD23 (BD Bioscience; \#553137) and streptavidin microbeads (Miltenyi Biotec; \#130-048-101) according to manufacturer's instructions. Cells were cultured for 2-3 d in RPMI 1640 medium (Corning) 
supplemented with non-essential amino acids (Corning), $2 \mathrm{mM}$ L-Glutamine (Corning), $25 \mathrm{mM}$ HEPES (pH 7.2-7.6), and $50 \mu \mathrm{M} \beta$-Mercaptoethanol and stimulated with $5 \mu \mathrm{g} / \mathrm{mL} \mathrm{F}\left(\mathrm{ab}^{\prime}\right)_{2}$ anti-mouse IgM (Jackson ImmunoResearch), $5 \mu \mathrm{g} /$ $\mathrm{mL}$ purified anti-mouse CD40 (BioXcell), and $50 \mathrm{ng} / \mathrm{mL}$ IL-21 (Peprotech). For thymocyte engulfment assays ${ }^{68}$ thymocytes were harvested and isolated from 4-6 week-old WT mice and treated with $50 \mu \mathrm{M}$ Dexamethasone for $4 \mathrm{~h}$ to induce apoptosis. Apoptotic thymocytes were then stained with $1 \mu \mathrm{M}$ CypHer5E (GE Healthcare) for $45 \mathrm{~min}$ at $37^{\circ} \mathrm{C}$ in serum-free Hank's Balanced Sodium Solution (HBSS). Stained thymocytes were co-cultured with splenocytes at a 1:10 splenocyte to thymocyte ratio. Apoptotic thymocytes were removed by washing with cold PBS and splenocytes were assessed for efferocytosis by flow cytometry. MHC-II

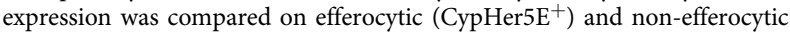
$\left(\right.$ CypHer5E $\left.{ }^{-}\right)$B cells.

Real-time RT-PCR and chromatin immunoprecipitation assays. Total RNA was isolated using the RNeasy Plus Mini kit (Qiagen; \#74134). cDNAs were prepared using the iScript cDNA synthesis kit (Biorad; \#1708841). Real-time PCR was performed using the iTaq Universal SYBR Green Supermix (Biorad; \#1725121). Gene expression was calculated using the $\Delta \Delta \mathrm{Ct}$ method and normalized to Ppia. For chromatin immunoprecipitation (ChIP) assays, B cell cultures were harvested at $\mathrm{d} 2$ and chromatin extracts were prepared using the truChIP Chromatin Shearing

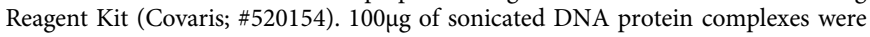
used for immunoprecipitations with anti-IRF5 (Abcam \#ab21689), anti-IRF8 (Cell Signaling; \#5628), or normal anti-rabbit Ig control antibodies. DNA purified from the immunoprecipitates and inputs was analyzed by qPCR. All primers are listed in Supplementary Table 2 .

ELISAs and antigen microarrays. For anti-dsDNA ELISA, plates were coated with $100 \mu \mathrm{g} / \mathrm{mL}$ salmon sperm DNA (Invitrogen; \#AM9680) at $37^{\circ} \mathrm{C}$ overnight and blocked in 2\% BSA in PBS at room temperature for $2 \mathrm{~h}$. For anti-cardiolipin and anti-phosphatidylserine ELISA, Immulon $2 \mathrm{HB}$ plates (Thermo) were coated with $75 \mu \mathrm{g} / \mathrm{mL}$ of cardiolipin or with $30 \mu \mathrm{g} / \mathrm{mL}$ phosphatidylserine dissolved in $100 \%$ ethanol overnight. Sera were diluted 1:200 and incubated on coated plates at $25^{\circ} \mathrm{C}$ for $2 \mathrm{~h}$. Supernatants from sorted ABCs were used neat after $7 \mathrm{~d}$ culture with $1 \mu \mathrm{M}$ Imiquimod. Plates were then incubated with HRP-labeled goat anti-mouse IgG or IgG2c Fc antibody for $1 \mathrm{~h}$ (eBioscience). $\mathrm{OD}_{450}$ was measured on a microplate reader. Autoantibody activities against a panel of autoantigen specificities were measured using an Autoantigen Microarray platform developed by the University of Texas Southwestern Medical Center ${ }^{69,70}$.

Histology and immunofluorescence staining. Tissue specimens were fixed in $10 \%$ neutral buffered formalin and embedded in paraffin. Tissue sections were stained with periodic acid-Schiff (PAS) or with hematoxylin and eosin (H\&E) and analyzed by light microscopy. The nephritis scoring system was adapted from the International Society of Nephrology/Renal Pathology Society (ISN/RPS) classification of human lupus nephritis. The final score accounted for morphological pattern (mesangial, capillary, membranous) and for the percentage of involved glomeruli. For immunofluorescence staining, kidneys or spleens were embedded in $\mathrm{OCT}$ and frozen in 2-methylbutane surrounded by dry ice. Frozen blocks were cut into $9 \mu \mathrm{m}$ section with cryotome and stored at $-80^{\circ} \mathrm{C}$. Upon thawing, sections were let dry at room temperature and stained. Spleen sections were stained with B220 (BD; RA3-6B2) and GL7 (BioLegend). Kidney sections were stained with FITC-labeled goat anti-mouse IgG (Jackson ImmunoResearch). Specimens were captured by Q capture software on a Nikon Eclipse microscope and quantifications were calculated using ImageJ software.

RNA-sequencing. Quality of all RNA and library preparations were evaluated with BioAnalyzer 2100 (Agilent). Sequencing libraries were sequenced by the Epigenomics Core Facility at Weill Cornell Medicine using a HiSeq2500, 50-bp singleend reads at a depth of $\sim 15-50$ million reads per sample. Read quality was assessed and adapters trimmed using FASTP ${ }^{71}$. Reads were then mapped to the mouse genome $(\mathrm{mm} 10)$ and reads in exons were counted against Gencode v27 with STAR2.6 Aligner ${ }^{72}$. Differential gene expression analysis was performed in R using edgeR3.24.3. Genes with low expression levels $(<2$ counts per million in at least one group) were filtered from all downstream analyses. Differential expression was estimated using quasi-likelihood framework. Benhamini-Hochberg false discovery rate (FDR) procedure was used to correct for multiple testing. Genes with an unadjusted $p$-value less than 0.01 were considered differentially expressed. Downstream analyses were performed in $\mathrm{R}$ using a visualization platform built with Shiny developed by bioinformaticians at the David Z. Rosensweig Genomics Research Center at HSS

Gene set enrichment analysis was performed using GSEA software (Broad Institute $)^{73}$. Genes were ranked by the difference of log-transformed count per million (cpm) for contrasted conditions. Molecular Signatures DataBase v7.0 (Broad Institute) was used as a source of gene sets with defined functional relevance. Gene sets were also curated from RNA-seq datasets of ABCs from female DKO mice and from the blood of SLE patients ${ }^{15,20}$ and from previously published $\mathrm{PB} / \mathrm{PC}$ dataset $^{74,75}$. Gene sets ranging between 15 and 2500 genes were included into the analysis. Nominal $p$-values were FDR corrected and gene sets with FDR $q<0.25$ were used to crease GSEA enrichment plots. Analyses of differentially expressed genes were also performed using the online webtool CPDB and upstream regulator analyses were conducted using the Enrichr databases ${ }^{76-78}$.

ATAC-sequencing. The nuclei of sorted $\mathrm{ABCs}$ from female $\mathrm{DKO}$, male $\mathrm{DKO}$, or Yaa-DKO mice were prepared by incubation of cells with nuclear preparation buffer $(0.30 \mathrm{M}$ sucrose, $10 \mathrm{mM}$ Tris pH 7.5, $60 \mathrm{mM} \mathrm{KCl,} 15 \mathrm{mM} \mathrm{NaCl}, 5 \mathrm{mM}$ $\mathrm{MgCl}_{2}, 0.1 \mathrm{mM}$ EGTA, $0.1 \% \mathrm{NP} 40,0.15 \mathrm{mM}$ spermine, $0.5 \mathrm{mM}$ spermidine, and 2 $\mathrm{mM} 6 \mathrm{AA})^{74}$. For library preparation ${ }^{79}$, fragments were amplified using $1 \times$ NEBnext PCR master mix and $1.25 \mu \mathrm{M}$ of custom Nextera PCR primers with the following PCR conditions: (1) $72^{\circ} \mathrm{C}$ for $5 \mathrm{~min}(2) 98^{\circ} \mathrm{C}$ for $30 \mathrm{~s}$ and (3) 3 cycles of $98^{\circ} \mathrm{C}$ for $10 \mathrm{~s}, 63^{\circ} \mathrm{C}$ for $30 \mathrm{~s}$, and $72^{\circ} \mathrm{C}$ for $1 \mathrm{~min}$. To reduce GC and size bias, the PCR reaction was monitored using $\mathrm{qPCR}$ in order to stop amplification before saturation. Additional amplification was performed for a total of 10-12 cycles. The libraries were purified using a Qiagen PCR cleanup kit yielding a final library concentration of $\sim 30 \mathrm{nM}$ in $20 \mu \mathrm{L}$. Paired-end 50 bp sequences were generated from samples with an Illumina HiSeq2500 and, following adapter trimming with FastP, were aligned against mouse genome $(\mathrm{mm} 10)$ using bowtie 2 with -local $-\mathrm{q}-\mathrm{p}$ options. Peaks were called with MACS2 with-macs2 callpeak - $f$ BAMPE-nomodel - shit -100-extsize 200-B-SPMR - $g$ \$GENOMESIZE - $q 0.01$ options. Peakassociated genes were defined based on the closest genes to these genomic regions using RefSeq co-ordinates of genes. We used the annotatePeaks command from HOMER to calculate ATAC-seq tag densities from different experiments and to create heatmaps of tag densities. Sequencing data were visualized by preparing custom tracks for the UCSC Genome browser. De novo transcription factor motif analysis was performed with motif finder program findMotifsGenome from HOMER package on ATAC-seq peaks. Peak sequences were compared to random genomic fragments of the same size and normalized G+C content to identify motifs enriched in the targeted sequences.

CUT\&RUN analysis. Freshly sorted ABCs and FoBs from both male and female DKO mice were used for the preparation of CUT\&RUN libraries as described in ${ }^{80}$ with slight modifications. We used three biological replicates for each condition. Sorted cells were immediately incubated overnight with H3K27me3 (anti- TriMethyl-Histone H3 (Lys27) (Cell Signaling Technology \#Rabbit mAb \#9733) or IgG isotype controls (Guinea Pig anti-Rabbit IgG $(\mathrm{H}+\mathrm{L})$ Secondary Antibody (novusbio \#NBP1-72763) at 1:50 dilution of antibody binding buffer with a final concentration of $0.05 \%$ digitonin. Following incubation and digestion CUTANA pAG-MNase from EpiCypher (SKU: 15-1116) the digested DNA fragments were release and purified by QIAquick PCR purification kit (\#2810). The resulting DNA fragments were end-repaired and barcoded libraries were made with the NEB Ultra II DNA Library Prep kit ((E7645S, E7335S \& E7500S)). The libraries were sequenced on Illumina NextSeq 500 to a minimum of 15 million reads per sample. The data was analyzed using CUT\&RUNTools pipeline ${ }^{81}$ and resulting peak calls from SECAR were used for subsequent analysis. The peaks from IgG isotype controls were excluded in the SECAR peak calling program. A master file of H3K27me3 peaks across all the conditions and replicated was created and ncbi/ BAMscale ${ }^{82}$ was used to quantify peaks and generate scaled coverage tracks for viewing in UCSC genome browser. The raw counts on each H3K27me3 peak were obtained using ncbi/BAMscale cov --bed master.H3K27me3.bed -prefix Peak_count -bam *.bam command and DESeq2 was employed to obtain differential H3K27me3 peaks between F-ABC and F-FOB mice. computeMatrix and plotHeap map functions were used from deepTools package to plot average tag densities and heatmap. Tag densities for the peaks in individual samples was calculated from DESeq2 and plotted as heatmap using Morpheus heatmap tool. The volcano plots were plotted with EnhancedVolcano ${ }^{83}$ available on Bioconductor and X chromosome heat of H3K27me3 signals was plotted using chromoMap. The z-statistic used in X chromosome H3K27me3 inactivation score heat map was obtained by implementing DESeq2 Wald test comparing F-ABC over F-FoB by taking log2 fold-change and dividing by its standard error resulting in z-statistic and a p-value is computed reporting that the probability that a $\mathrm{z}$-statistic is small enough to reject null hypothesis. We summarized the differential peaks obtained by DESeq2 comparisions between F-ABC, F-FoB, M-ABC and M-FoB in Supplementary Data 3.

IgH sequencing and immune repertoire analysis. Genomic DNA was extracted from sorted cells using the QIAGEN Gentra DNA purification kit (Qiagen, \#158689). Primer sequencies and library preparation were described previously ${ }^{9}$. Samples were amplified in duplicate (2 biological replicates per sample) using $100 \mathrm{ng}$ of input DNA per replicate (Supplementary Data 4). Sequencing was performed on an Illumina MiSeq instrument in the Human Immunology Core facility at the University of Pennsylvania using a 2x300bp paired-end kit. Sequencing data analysis was also previously described ${ }^{9}$. Sequences were quality controlled with pRESTO $^{84}$. Briefly, paired reads were aligned, sequences with low quality scores were discarded, and base calls with low confidence were masked with "N"s. IgBLAST was then used to align sequences to $\mathrm{V}$ - and J-genes in the IMGT database ${ }^{85}$. To group related sequences together into clones, ImmuneDB hierarchically clusters sequences with the same VH gene, same JH gene, same CDR3 length, and $85 \%$ identity at the amino acid level within the CDR3 sequence ${ }^{86}$. Clones with consensus CDR3 sequences within 2 
nucleotides of each other were further collapsed to account for incorrect gene calls. Sequencing data were submitted to SRA under project number PRJNA663307 in accordance with the MiAIRR standard ${ }^{87}$. Prism v8.4.3 was used for D20, Jaccard index, SHM, CDR3 length, and VH gene usage histogram plots. Morpheus (Broad Institute) was used for VH heatmap. Venn Diagrams were generated in (http://www. interactivenn.net/). Other calculations were performed as described previously ${ }^{86}$.

Statistics. All plots show datapoints from independent mice pooled across multiple experiments, unless otherwise noted. $p$-values were calculated with two-tailed $t$-tests or ANOVA followed by multi-group comparisons, as indicated in the figure legends. Survival data was tested by Kaplan-Meyer analysis with significance determined by the log-rank (Mantel-Cox) test. Correlation data was tested by paired Pearson correlations. $p$-values of $<0.05$ were considered significant. Ns: not significant, ${ }^{*} p<0.05,{ }^{* *} p<0.01,{ }^{* * *} p<0.001,{ }^{* * * *} p<0.0001$. Statistical analysis was performed with Graphpad Prism 8.

Reporting summary. Further information on research design is available in the Nature Research Reporting Summary linked to this article.

\section{Data availability}

Sequence data that support the findings of this study have been deposited in NCBI's Gene Expression Omnibus (GEO) with the primary accession code GSE175365 and to SRA under project number PRJNA663307. Other reagents and materials are available upon reasonable request from the corresponding author. Source data are provided with this paper.

Received: 15 October 2020; Accepted: 15 July 2021; Published online: 10 August 2021

\section{References}

1. Scully, E. P., Haverfield, J., Ursin, R. L., Tannenbaum, C. \& Klein, S. L. Considering how biological sex impacts immune responses and COVID-19 outcomes. Nat. Rev. Immunol. 20, 442-447 (2020).

2. Liu, Z. \& Davidson, A. Taming lupus-a new understanding of pathogenesis is leading to clinical advances. Nat. Med. 18, 871-882 (2012).

3. Rubtsova, K., Marrack, P. \& Rubtsov, A. V. Sexual dimorphism in autoimmunity. J. Clin. Invest. 125, 2187-2193 (2015)

4. Souyris M, et al. TLR7 escapes X chromosome inactivation in immune cells. Sci. Immunol. 3, eaap8855 (2018)

5. Hao, Y., O’Neill, P., Naradikian, M. S., Scholz, J. L. \& Cancro, M. P. A B-cell subset uniquely responsive to innate stimuli accumulates in aged mice. Blood 118, 1294-1304 (2011)

6. Rubtsov, A. V. et al. Toll-like receptor 7 (TLR7)-driven accumulation of a novel CD11c $(+)$ B-cell population is important for the development of autoimmunity. Blood 118, 1305-1315 (2011).

7. Jenks, S. A. et al. Distinct effector B cells induced by unregulated toll-like receptor 7 contribute to pathogenic responses in systemic lupus erythematosus. Immunity 49, 725-739 e726 (2018).

8. Cancro, M. P. Age-associated B cells. Annu. Rev. Immunol. 38, 315-340 (2020).

9. Johnson, J. L. et al. The transcription factor T-bet resolves memory B cell subsets with distinct tissue distributions and antibody specificities in mice and humans. Immunity 52, 842-855 e846 (2020).

10. Kenderes, K. J. et al. T-Bet(+) IgM memory cells generate multi-lineage effector B cells. Cell Rep. 24, 824-837 e823 (2018).

11. Austin, J. W. et al. Overexpression of T-bet in HIV infection is associated with accumulation of B cells outside germinal centers and poor affinity maturation. Sci. Transl. Med. 11, eaax0904 (2019).

12. Woodruff, M. C. et al. Extrafollicular B cell responses correlate with neutralizing antibodies and morbidity in COVID-19. Nat. Immunol. 21, 1506-1516 (2020).

13. Kaneko, N. et al. Loss of Bcl-6-expressing $\mathrm{T}$ follicular helper cells and germinal centers in COVID-19. Cell 183, 143-157 e113 (2020).

14. Jenks, S. A., Cashman, K. S., Woodruff, M. C., Lee, F. E. \& Sanz, I. Extrafollicular responses in humans and SLE. Immunol. Rev. 288, 136-148 (2019).

15. Wang, S. et al. IL-21 drives expansion and plasma cell differentiation of autoreactive CD11c(hi)T-bet(+) B cells in SLE. Nat. Commun. 9, 1758 (2018).

16. Arazi, A. et al. The immune cell landscape in kidneys of patients with lupus nephritis. Nat. Immunol. 20, 902-914 (2019).

17. Rubtsova, K. et al. B cells expressing the transcription factor T-bet drive lupuslike autoimmunity. J. Clin. Invest 127, 1392-1404 (2017).

18. Du, S. W., Arkatkar, T., Jacobs, H. M., Rawlings, D. J. \& Jackson, S. W. Generation of functional murine $\mathrm{CD} 11 \mathrm{c}(+)$ age-associated $\mathrm{B}$ cells in the absence of B cell T-bet expression. Eur. J. Immunol. 49, 170-178 (2019).
19. Levack, R. C., Newell, K. L., Popescu, M., Cabrera-Martinez, B. \& Winslow, G. M. CD11c(+) T-bet(+) B cells require IL-21 and IFN-gamma from type $1 \mathrm{~T}$ follicular helper cells and intrinsic Bcl-6 expression but develop normally in the absence of T-bet. J. Immunol. 205, 1050-1058 (2020).

20. Manni, M. et al. Regulation of age-associated B cells by IRF5 in systemic autoimmunity. Nat. Immunol. 19, 407-419 (2018).

21. Manni, M., Ricker, E. \& Pernis, A. B. Regulation of systemic autoimmunity and CD11c(+) Tbet(+) B cells by SWEF proteins. Cell Immunol. 321, 46-51 (2017).

22. Biswas, P. S. et al. Dual regulation of IRF4 function in $\mathrm{T}$ and $\mathrm{B}$ cells is required for the coordination of T-B cell interactions and the prevention of autoimmunity. J. Exp. Med. 209, 581-596 (2012).

23. Becart, S. \& Altman, A. SWAP-70-like adapter of T cells: a novel Lckregulated guanine nucleotide exchange factor coordinating actin cytoskeleton reorganization and $\mathrm{Ca} 2+$ signaling in T cells. Immunol. Rev. 232, 319-333 (2009).

24. Shinohara, M. et al. SWAP-70 is a guanine-nucleotide-exchange factor that mediates signalling of membrane ruffling. Nature 416, 759-763 (2002).

25. Skibinski, D. A. G. et al. Induction of human T-cell and cytokine responses following vaccination with a novel influenza vaccine. Sci. Rep. 8, 18007 (2018).

26. Tran, T. M. et al. A molecular signature in blood reveals a role for p53 in regulating malaria-induced inflammation. Immunity 51, 750-765 e710 (2019).

27. Kwon, Y. C. et al. Genome-wide association study in a Korean population identifies six novel susceptibility loci for rheumatoid arthritis. Ann. Rheum. Dis. 79, 1438-1445 (2020).

28. Zheng, Q., Ma, Y., Chen, S., Che, Q. \& Chen, D. The integrated landscape of biological candidate causal genes in coronary artery disease. Front Genet 11, 320 (2020).

29. Sun, C. et al. High-density genotyping of immune-related loci identifies new SLE risk variants in individuals with Asian ancestry. Nat. Genet 48, 323-330 (2016).

30. Langefeld, C. D. et al. Transancestral mapping and genetic load in systemic lupus erythematosus. Nat. Commun. 8, 16021 (2017).

31. Serwas, N. K. et al. Human DEF6 deficiency underlies an immunodeficiency syndrome with systemic autoimmunity and aberrant CTLA-4 homeostasis. Nat. Commun. 10, 3106 (2019).

32. Fournier, B. et al. DEF6 deficiency, a mendelian susceptibility to EBV infection, lymphoma, and autoimmunity. J. Allergy Clin. Immunol. 147, 740-743 e749 (2021).

33. Deane, J. A. et al. Control of toll-like receptor 7 expression is essential to restrict autoimmunity and dendritic cell proliferation. Immunity 27, 801-810 (2007).

34. Rosenfeld, A. M. et al. Computational evaluation of B-cell clone sizes in bulk populations. Front Immunol. 9, 1472 (2018).

35. Scharer, C. D. et al. Epigenetic programming underpins B cell dysfunction in human SLE. Nat. Immunol. 20, 1071-1082 (2019).

36. Yu, F., Sharma, S., Edwards, J., Feigenbaum, L. \& Zhu, J. Dynamic expression of transcription factors T-bet and GATA-3 by regulatory T cells maintains immunotolerance. Nat. Immunol. 16, 197-206 (2015).

37. Chiche, L. et al. Modular transcriptional repertoire analyses of adults with systemic lupus erythematosus reveal distinct type I and type II interferon signatures. Arthritis Rheumatol. 66, 1583-1595 (2014).

38. Robinson, M. J. et al. The amount of BCL6 in B cells shortly after antigen engagement determines their representation in subsequent germinal centers. Cell Rep. 30, 1530-1541 e1534 (2020).

39. Zhang, T. T. et al. Germinal center B cell development has distinctly regulated stages completed by disengagement from T cell help. Elife 6, e19552 (2017).

40. Rivera-Correa, J. et al. Atypical memory B-cells are associated with Plasmodium falciparum anemia through anti-phosphatidylserine antibodies. Elife 8, e48309 (2019).

41. Barrat, F. J., Crow, M. K. \& Ivashkiv, L. B. Interferon target-gene expression and epigenomic signatures in health and disease. Nat. Immunol. 20, 1574-1583 (2019).

42. Schneider, W. M., Chevillotte, M. D. \& Rice, C. M. Interferon-stimulated genes: a complex web of host defenses. Annu Rev. Immunol. 32, 513-545 (2014).

43. Nehar-Belaid, D. et al. Mapping systemic lupus erythematosus heterogeneity at the single-cell level. Nat. Immunol. 21, 1094-1106 (2020).

44. Kyogoku, C. et al. Cell-specific type I IFN signatures in autoimmunity and viral infection: what makes the difference? PLoS ONE 8, e83776 (2013).

45. Kotliarov, Y. et al. Broad immune activation underlies shared set point signatures for vaccine responsiveness in healthy individuals and disease activity in patients with lupus. Nat. Med 26, 618-629 (2020).

46. Hadjadj, J. et al. Impaired type I interferon activity and inflammatory responses in severe COVID-19 patients. Science 369, 718-724 (2020).

47. Antonczyk, A. et al. Direct inhibition of IRF-dependent transcriptional regulatory mechanisms associated with disease. Front Immunol. 10, 1176 (2019) 
48. Jensen, M. A. \& Niewold, T. B. Interferon regulatory factors: critical mediators of human lupus. Transl. Res. 165, 283-295 (2015).

49. Ricker, E. et al. Serine-threonine kinase ROCK2 regulates germinal center B cell positioning and cholesterol biosynthesis. J. Clin. Invest 130, 3654-3670 (2020).

50. Wang, H. et al. Transcription factors IRF8 and PU.1 are required for follicular B cell development and BCL6-driven germinal center responses. Proc. Natl Acad. Sci. USA 116, 9511-9520 (2019).

51. Aranburu, A. et al. Age-associated B cells expanded in autoimmune mice are memory cells sharing H-CDR3-selected repertoires. Eur. J. Immunol. 48, 509-521 (2018).

52. Trivedi, N. et al. Liver is a generative site for the B cell response to Ehrlichia muris. Immunity 51, 1088-1101 e1085 (2019).

53. Arnold, A. P., Cassis, L. A., Eghbali, M., Reue, K. \& Sandberg, K. Sex hormones and sex chromosomes cause sex differences in the development of cardiovascular diseases. Arterioscler Thromb. Vasc. Biol. 37, 746-756 (2017).

54. Strassheim, D. et al. RhoGTPase in vascular disease. Cells 8, 551 (2019).

55. Yu, B. et al. B cell-specific XIST complex enforces X-inactivation and restrains atypical B cells. Cell 184, 1790-1803 (2021).

56. Moulton, V. R. Sex hormones in acquired immunity and autoimmune disease. Front Immunol. 9, 2279 (2018).

57. Zhao, R. et al. A GPR174-CCL21 module imparts sexual dimorphism to humoral immunity. Nature 577, 416-420 (2020).

58. Wilk, A. J. et al. A single-cell atlas of the peripheral immune response in patients with severe COVID-19. Nat. Med. 26, 1070-1076 (2020).

59. Mathew, D. et al. Deep immune profiling of COVID-19 patients reveals distinct immunotypes with therapeutic implications. Science 369, eabc8511 (2020).

60. Rodriguez $\mathrm{Y}$, et al. Autoinflammatory and autoimmune conditions at the crossroad of COVID-19. J. Autoimmun. 114, 102506 (2020).

61. Lucas, C. et al. Longitudinal analyses reveal immunological misfiring in severe COVID-19. Nature 584, 463-469 (2020).

62. Camell, C. D. et al. Aging induces an Nlrp3 inflammasome-dependent expansion of adipose B cells that impairs metabolic homeostasis. Cell Metab. 30, 1024-1039 e1026 (2019)

63. Xia, Y. et al. The mevalonate pathway is a druggable target for vaccine adjuvant discovery. Cell 175, 1059-1073 e1021 (2018).

64. Lund, J. M. et al. Recognition of single-stranded RNA viruses by Toll-like receptor 7. Proc. Natl Acad. Sci. USA 101, 5598-5603 (2004).

65. Izui, S. et al. The Yaa gene model of systemic lupus erythematosus. Immunol. Rev. 144, 137-156 (1995).

66. Chandrasekaran, U. et al. Regulation of effector Treg cells in murine lupus. Arthritis Rheumatol. 68, 1454-1466 (2016).

67. Kwon, K. et al. Instructive role of the transcription factor E2A in early B lymphopoiesis and germinal center B cell development. Immunity 28, 751-762 (2008).

68. Perry, J. S. A. et al. Interpreting an apoptotic corpse as anti-inflammatory involves a chloride sensing pathway. Nat. Cell Biol. 21, 1532-1543 (2019).

69. Li, Q. Z. et al. Identification of autoantibody clusters that best predict lupus disease activity using glomerular proteome arrays. J. Clin. Invest 115, 3428-3439 (2005).

70. Li, Q. Z. et al. Protein array autoantibody profiles for insights into systemic lupus erythematosus and incomplete lupus syndromes. Clin. Exp. Immunol 147, 60-70 (2007).

71. Chen, S., Zhou, Y., Chen, Y. \& Gu, J. fastp: an ultra-fast all-in-one FASTQ preprocessor. Bioinformatics 34, i884-i890 (2018).

72. Dobin, A. et al. STAR: ultrafast universal RNA-seq aligner. Bioinformatics 29, 15-21 (2013)

73. Subramanian, A. et al. Gene set enrichment analysis: a knowledge-based approach for interpreting genome-wide expression profiles. Proc. Natl Acad. Sci. USA 102, 15545-15550 (2005).

74. Minnich, M. et al. Multifunctional role of the transcription factor Blimp-1 in coordinating plasma cell differentiation. Nat. Immunol. 17, 331-343 (2016).

75. Low, M. S. Y. et al. IRF4 activity is required in established plasma cells to regulate gene transcription and mitochondrial homeostasis. Cell Rep. 29, 2634-2645 e2635 (2019).

76. Chen, E. Y. et al. Enrichr: interactive and collaborative HTML5 gene list enrichment analysis tool. BMC Bioinformatics 14, 128 (2013).

77. Kuleshov, M. V. et al. Enrichr: a comprehensive gene set enrichment analysis web server 2016 update. Nucleic Acids Res. 44, W90-W97 (2016).

78. Kamburov, A., Stelzl, U., Lehrach, H. \& Herwig, R. The ConsensusPathDB interaction database: 2013 update. Nucleic Acids Res. 41, D793-D800 (2013).

79. Buenrostro, J. D., Giresi, P. G., Zaba, L. C., Chang, H. Y. \& Greenleaf, W. J. Transposition of native chromatin for fast and sensitive epigenomic profiling of open chromatin, DNA-binding proteins and nucleosome position. Nat. Methods 10, 1213-1218 (2013).
80. Skene, P. J., Henikoff, J. G. \& Henikoff, S. Targeted in situ genome-wide profiling with high efficiency for low cell numbers. Nat. Protoc. 13, 1006-1019 (2018).

81. Zhu, Q., Liu, N., Orkin, S. H. \& Yuan, G. C. CUT\&RUNTools: a flexible pipeline for CUT\&RUN processing and footprint analysis. Genome Biol. 20, 192 (2019).

82. Pongor, L. S. et al. BAMscale: quantification of next-generation sequencing peaks and generation of scaled coverage tracks. Epigenetics Chromatin 13, 21 (2020).

83. Blighe K, Rana S, Lewis M. EnhancedVolcano: Publication-ready volcano plots with enhanced colouring and labeling. R package version 1.8.0. (2020)

84. Vander Heiden, J. A. et al. pRESTO: a toolkit for processing high-throughput sequencing raw reads of lymphocyte receptor repertoires. Bioinformatics $\mathbf{3 0}$, 1930-1932 (2014)

85. Giudicelli, V., Chaume, D. \& Lefranc, M. P. IMGT/GENE-DB: a comprehensive database for human and mouse immunoglobulin and $\mathrm{T}$ cell receptor genes. Nucleic Acids Res. 33, D256-D261 (2005).

86. Meng, W. et al. An atlas of B-cell clonal distribution in the human body. Nat. Biotechnol. 35, 879-884 (2017).

87. Rubelt, F. et al. Adaptive immune receptor repertoire community recommendations for sharing immune-repertoire sequencing data. Nat. Immunol. 18, 1274-1278 (2017).

88. Salameh, T. J. et al. A supervised learning framework for chromatin loop detection in genome-wide contact maps. Nat. Commun. 11, 3428 (2020).

89. Rao, S. S. et al. A 3D map of the human genome at kilobase resolution reveals principles of chromatin looping. Cell 159, 1665-1680 (2014).

\section{Acknowledgements}

We thank members of the HSS Research Institute for thoughtful discussions and reagents. This work was supported by the US National Institutes of Health (AR064883 and AR070146 to ABP; T32 Rheumatology Research Training Grant to E.R.; P30 CA016520 and P30 AI0450080 to E.L.P.), the Rheumatology Research Foundation, the Lupus Research Alliance, the Peter Jay Sharp Foundation, the Tow Foundation which provided support for the David Z. Rosensweig Genomics Research Center, Giammaria Giuliani and the Ambrose Monell Foundation, the Barbara Volcker Center for the Michael D. Lockshin Fellowship (M.M.), and Marina Kellen French and the Anna-Maria and Stephen Kellen Foundation (D.J. and P.S.). Technical support was provided by the Epigenomics Core, the Microscopy and Imaging Core, and the Flow Cytometry Core Facility of Weill Cornell Medicine, by the Laboratory of Comparative Pathology at Memorial Sloan Kettering Cancer Center, by the Human Immunology Core at the Perelman School of Medicine, and by the Genomics and Microarray Core Facility at the University of Texas Southwestern Medical Center, and from the Office of the Director of the National Institutes of Health under Award Number S10OD019986 to Hospital for Special Surgery.

\section{Author contributions}

E.R. designed and performed the experiments, interpreted the experiments, and wrote the manuscript; M.M. designed and performed the experiments and interpreted the experiments; D.F.C., D.J., S.G., and J.R.C. performed the experiments; W.M., A.R., and E.L.P. conducted the IgH sequencing bioinformatics analyses; T.P. assisted with the histological analyses; M.B. performed the CUT\&RUN bioinformatics analyses; Y.C. performed the RNA-seq and ATAC-seq bioinformatics analyses; P.K.S. helped write the manuscript; R.J. generated the Swap $70^{-I-}$ mice and helped write the manuscript; A.B.P. designed and supervised the study, interpreted the experiments, and wrote the manuscript.

\section{Competing interests}

The authors declare no competing interests.

\section{Additional information}

Supplementary information The online version contains supplementary material available at https://doi.org/10.1038/s41467-021-25102-8.

Correspondence and requests for materials should be addressed to A.B.P.

Peer review information Nature Communications thanks Amr Sawalha, George Tsokos and the other, anonymous, reviewer(s) for their contribution to the peer review of this work. Peer reviewer reports are available.

Reprints and permission information is available at http://www.nature.com/reprints

Publisher's note Springer Nature remains neutral with regard to jurisdictional claims in published maps and institutional affiliations. 
(c) (i) Open Access This article is licensed under a Creative Commons Attribution 4.0 International License, which permits use, sharing, adaptation, distribution and reproduction in any medium or format, as long as you give appropriate credit to the original author(s) and the source, provide a link to the Creative Commons license, and indicate if changes were made. The images or other third party material in this article are included in the article's Creative Commons license, unless indicated otherwise in a credit line to the material. If material is not included in the article's Creative Commons license and your intended use is not permitted by statutory regulation or exceeds the permitted use, you will need to obtain permission directly from the copyright holder. To view a copy of this license, visit http://creativecommons.org/ licenses/by/4.0/.

(C) The Author(s) 2021 